\title{
Impact of hadronic and nuclear corrections on global analysis of spin-dependent parton distributions
}

\author{
P. Jimenez-Delgado ${ }^{1}$, A. Accardi ${ }^{1,2}$, W. Melnitchouk ${ }^{1}$ \\ ${ }^{1}$ Jefferson Lab, Newport News, Virginia 23606, USA \\ ${ }^{2}$ Hampton University, Hampton, Virginia 23668, USA \\ Jefferson Lab Angular Momentum (JAM) Collaboration
}

(Dated: November 8, 2018)

\begin{abstract}
We present the first results of a new global next-to-leading order analysis of spin-dependent parton distribution functions from the most recent world data on inclusive polarized deep-inelastic scattering, focusing in particular on the large- $x$ and low- $Q^{2}$ regions. By directly fitting polarization asymmetries we eliminate biases introduced by using polarized structure function data extracted under nonuniform assumptions for the unpolarized structure functions. For analysis of the large- $x$ data we implement nuclear smearing corrections for deuterium and ${ }^{3} \mathrm{He}$ nuclei, and systematically include target mass and higher twist corrections to the $g_{1}$ and $g_{2}$ structure functions at low $Q^{2}$. We also explore the effects of $Q^{2}$ and $W^{2}$ cuts in the data sets, and the potential impact of future data on the behavior of the spin-dependent parton distributions at intermediate and large $x$.
\end{abstract}




\section{INTRODUCTION}

The decomposition of the nucleon's spin into its constituent quark and gluon components remains one of the most important challenges in nuclear and particle physics. Significant progress on this problem has been made over the last 25 years, since the early polarized deepinelastic scattering (DIS) experiments [1] indicated that quarks carry only a small fraction of the proton's spin. In the intervening years a number of dedicated experimental programs have been undertaken at various accelerator facilities worldwide, refining our knowledge of the spin distributions through measurements of polarization asymmetries in inclusive and semi-inclusive lepton-nucleon scattering and polarized proton-proton collisions (for recent reviews, see Refs. [2-4]).

On the theoretical front, the discussions about quark and gluon (or parton) contributions to the nucleon spin have led to a better understanding of fundamental questions related to gauge invariance of spin-dependent matrix elements of quark and gluon operators, and the appropriate definitions of parton orbital angular momentum in QCD (see Ref. [5] and references therein). Independent of their physical interpretation, extraction of spin-dependent parton distribution functions (PDFs) is an important phenomenological pursuit, in which one seeks a consistent description of data from a variety of experiments within a perturbative QCD framework. With the growing body of experimental data being accumulated, and plans for future experiments at RHIC, COMPASS and Jefferson Lab to achieve greater precision and access to regions of kinematics hitherto unexplored, the need exists to develop the theoretical tools necessary to optimally utilize the new empirical information.

Systematic studies of spin-dependent PDFs have been performed by a number of collaborations, with next-to-leading order (NLO) analyses using the standard global fitting methodology undertaken by the DSSV (de Florian, Sassot, Stratmann and Vogelsang) [6], LSS (Leader, Sidorov and Stamenov) [7], BB (Blümlein and Böttcher) [8], and AAC (Asymmetry Analysis Collaboration) [9] groups. All of these analyses utilize inclusive DIS data on proton, deuteron and ${ }^{3} \mathrm{He}$ targets, while the LSS10 [7] and DSSV09 [6] PDFs are also constrained by semi-inclusive DIS data. The DSSV09 group uses in addition the polarized $p p$ scattering data at NLO, while the AAC [9] fits these data via a $K$-factor approximation for the NLO corrections. The more recent NNPDF distributions [10] are based on a neural network approach, while the parametrizations from Ref. [11] are inspired by a statistical 
model. A new NLO analysis of inclusive and semi-inclusive DIS data was also recently performed in Ref. [12].

Determining the nucleon spin fractions carried by quarks and gluons requires integrating the spin PDFs over all values of the momentum fraction $x$. The small- $x$ region in particular contributes significantly to the integrals (or lowest moments) of the distributions, and considerable effort has been made to understand the spin PDFs in this region both experimentally and theoretically. On the other hand, at large values of $x$ the $u$ and $d$ quark PDFs are very sensitive to the dynamics underlying the breaking of $\mathrm{SU}(2) \times \mathrm{SU}(2)$ spin-flavor symmetry [13-15], as well as to the presence of quark orbital angular momentum in the nucleon [16]. Unfortunately, PDFs are notoriously difficult to extract at high values of $x$ due to the rapidly dropping cross sections in this region, and consequently spin structure measurements have received relatively little attention for $x \gtrsim 0.5$.

More recently, experiments at Jefferson Lab utilizing the high lumonisities and polarized beams available with the CEBAF accelerator have collected high-precision data on polarization asymmetries and cross sections for both longitudinally and transversely polarized targets [17]. The new data provide a unique, if somewhat limited, window on the high- $x$ region, which will be further extended following the $12 \mathrm{GeV}$ energy upgrade [18]. In an effort to maximally exploit the new data, the Jefferson Lab Angular Momentum (JAM) Collaboration [19] has embarked on a program to perform a global NLO analysis of world data, over a large range of kinematics, including a systematic study of spin-dependent PDFs in the high- $x$ and low- $Q^{2}$ region.

From the kinematics of inclusive DIS, at fixed four-momentum transfer squared $Q^{2}$, increasing values of $x$ are naturally correlated with decreasing invariant masses $W$ of the produced hadronic system, $W^{2}=M^{2}+Q^{2}(1-x) / x$, where $M$ is the mass of the nucleon. To ensure that the leading twist contribution dominates the scattering process, one must restrict $W$ to be large enough for the final state to be generated mostly by incoherent fragmentation of partons into hadrons, with $W$ above the region where coherent resonance structures are visible, $W \geq W_{\text {res }} \approx(1.8-2.0) \mathrm{GeV}$. At fixed $Q^{2}$ this means that $x$ is bounded by $x \leq x_{\text {res }}=Q^{2} /\left(W_{\text {res }}^{2}-M^{2}+Q^{2}\right)$, so that in practice at $Q^{2}=1 \mathrm{GeV}^{2}$ the DIS region is limited to $x \lesssim 0.25$, and even at $Q^{2}=5 \mathrm{GeV}^{2}$ it is constrained to $x \lesssim 0.6$. Since the experimentally explored $Q^{2}$ range is more restricted in polarized DIS than in unpolarized DIS, a significantly larger portion of the spin-dependent data lie in the small- $Q^{2}$ region. Con- 
sequently, obtaining direct information on PDFs at large $x$ presents an even more difficult task than for spin-averaged PDFs, which themselves have sizeable uncertainties for $x \gtrsim 0.7$ [20]. To compensate for the smaller overall number of data points available to global spin PDF analyses, one is then typically forced to make use of data down to $Q^{2} \approx 1 \mathrm{GeV}^{2}$.

The inclusion of data at large $x$ (or low $W$ ) and low $Q^{2}$ presents additional challenges for global PDF studies. To account for deviations in the low- $Q^{2}$ data from the logarithmic $Q^{2}$ dependence expected from perturbative QCD, corrections from various nonperturbative effects at finite $Q^{2}$, such as target mass and higher twist corrections, must be included. Several of the existing analyses implement some of these effects, such as the target mass corrections (TMCs) in Refs. [7, 8, 10], and higher twist corrections to the $g_{1}$ structure function in the LSS10 [7] and BB10 [8] fits. In the present analysis we incorporate both twist-3 and twist-4 corrections to $g_{1}$, as well as a twist-3 correction to the $g_{2}$ structure function.

Moreover, when using data obtained from experiments with polarized deuterium or ${ }^{3} \mathrm{He}$ nuclei, whose use is necessary for the separation of the $u$ and $d$ quark flavors, nuclear effects must be removed. While existing analyses typically do account for some nuclear effects through the method of effective polarizations, at large values of $x$ nuclear Fermi motion plays an increasingly important role, requiring nuclear smearing corrections to be applied, as well as other possible corrections associated with nucleon off-shell and non-nucleonic degrees of freedom [21]. The nuclear corrections have been shown in recent unpolarized PDF analyses [20, 22-27] to be essential for correctly describing DIS data at large $x$ and low $W$, and are even more important in polarized fits, where low- $W$ DIS data comprise a substantial fraction of the available data. In this work we consistently apply the nuclear smearing corrections to both the $g_{1}$ and $g_{2}$ structure functions for both deuterium and ${ }^{3} \mathrm{He}$, within the framework of the weak binding approximation [21, 28, 29], and examine their impact on the extracted PDFs.

The strategy adopted in most previous global PDF studies has involved fitting the $g_{1}$ structure function or the polarization asymmetry $A_{1}$, which are typically extracted from the experimental longitudinal polarization asymmetry using parametrizations of the unpolarized structure functions and assumptions about the transverse spin dependent $g_{2}$ structure function. In contrast, the JAM analysis directly fits the measured longitudinal and transverse asymmetries, where available, thereby eliminating any potential biases introduced into 
the analysis by the use of inconsistent unpolarized structure function inputs obtained from separate experimental analyses. The unpolarized PDFs used in the JAM analysis represent a new global fit to the world's spin-averaged data, performed along the lines of the spindependent fit and using a similar set of assumptions, kinematic cuts, and theoretical nuclear and finite- $Q^{2}$ corrections (for details see Ref. [27]).

With the focus of this study being primarily on PDFs at intermediate and large $x$, our strategy will be to first systematically explore the influence of the various corrections on the $u$ and $d$ quark distributions, whose determination in principle requires only inclusive DIS data. Once the basic fits are established, in subsequent work the JAM analysis will be extended to study sea quark flavor decomposition and gluon polarization at small $x$ using semi-inclusive DIS and polarized hadron-hadron scattering data.

The outline of this paper is as follows. In Sec. II we summarize the basic formulas for the inclusive DIS cross sections and asymmetries, and the polarized data sets used in this analysis. The salient features of the theoretical QCD framework are reviewed in Sec. III, including the choice of PDF parametrization and the analysis of PDF errors. Here we also present the reference fit which will be used as the baseline for the subsequent study of the various strong-interaction effects discussed in the rest of the paper. The effects of the nuclear smearing corrections in the deuteron and ${ }^{3} \mathrm{He}$ data on the fitted PDFs are described in Sec. IV, and those due to finite- $Q^{2}$ corrections in Sec. V. The results of the full JAM fit are presented in Sec. VI, and parameter values for the leading twist and higher twist contributions to the structure functions are provided. The impact of the recent highprecision data from Jefferson Lab at low $W$ and $Q^{2}$ is discussed in Sec. VII, together with the effects of different kinematic cuts in $W$ and $Q^{2}$ on the determination of the leading twist PDFs and higher twist corrections. Finally, in Sec. VIII we recount the findings of the present study, and outline future plans for the JAM PDF analysis.

\section{POLARIZED DEEP-INELASTIC SCATTERING}

In this section we briefly review the definitions of the inclusive DIS cross sections and polarization asymmetries that will provide the data to be fitted in the JAM analysis. We utilize data on both the longitudinal and transverse asymmetries for hydrogen, deuterium and ${ }^{3} \mathrm{He}$ targets, or on the $A_{1}$ and $A_{2}$ asymmetries where these are given instead. 


\section{A. Cross sections and asymmetries}

Most of the information that currently exists on the partonic spin structure of the nucleon has come from experiments involving inclusive scattering of longitudinally polarized leptons from longitudinally polarized targets. By taking differences of cross sections with the spin of the target parallel and antiparallel to that of the lepton, one measures primarily the $g_{1}$ structure function of the nucleon, which in the parton model is expressed in terms of the spin (or helicity) dependent PDFs. Information on the $g_{2}$ structure function, which does not have a simple partonic interpretation, can be gathered by measuring differences of cross sections with the target polarized transversely to the beam polarization.

The asymmetries for longitudinal $\left(A_{\|}\right)$and transverse $\left(A_{\perp}\right)$ target polarization are defined in terms of the differential cross sections $\sigma \equiv d^{2} \sigma / d \Omega d E^{\prime}$ by

$$
A_{\|}=\frac{\sigma^{\uparrow \Downarrow}-\sigma^{\uparrow \Uparrow}}{\sigma^{\uparrow \Downarrow}+\sigma^{\uparrow \Uparrow}}, \quad A_{\perp}=\frac{\sigma^{\uparrow \Rightarrow}-\sigma^{\uparrow \Leftarrow}}{\sigma^{\uparrow \Rightarrow}+\sigma^{\uparrow \Leftarrow}},
$$

where the arrows $\uparrow$ and $\Uparrow(\Downarrow)$ denote the electron and nucleon spins in the same (opposite) directions, respectively, with the corresponding nucleon polarizations transverse to the beam direction labeled by $\Rightarrow$ and $\Leftarrow$. It is convenient also to define the spin asymmetries $A_{1}$ and $A_{2}$, such that

$$
A_{\|}=D\left(A_{1}+\eta A_{2}\right), \quad A_{\perp}=d\left(A_{2}-\xi A_{1}\right),
$$

where the kinematical variables are given by

$$
\begin{gathered}
D=\frac{y(2-y)\left(2+\gamma^{2} y\right)}{2\left(1+\gamma^{2}\right) y^{2}+\left(4(1-y)-\gamma^{2} y^{2}\right)(1+R)}, \quad \eta=\gamma \frac{4(1-y)-\gamma^{2} y^{2}}{(2-y)\left(2+\gamma^{2} y\right)}, \\
d=\frac{\sqrt{4(1-y)-\gamma^{2} y^{2}}}{2-y} D, \quad \xi=\gamma \frac{2-y}{2+\gamma^{2} y} .
\end{gathered}
$$

Here $y=\nu / E=Q^{2} / 2 M x E$ is fraction of the incident lepton's energy $E$ transferred to the target, $\nu$ and $Q^{2}$ are the energy transfer and four-momentum transfer squared (virtuality of the photon), respectively, and $x=Q^{2} / 2 M \nu$ is the Bjorken scaling variable, with $M$ the nucleon mass, and $\gamma^{2}=4 M^{2} x^{2} / Q^{2}$. The function $R$ is the ratio of cross sections for longitudinal to transversely polarized virtual photons,

$$
R=\frac{F_{L}}{\left(1+\gamma^{2}\right) F_{2}-F_{L}}
$$


with the longitudinal structure function $F_{L}$ defined in terms of the unpolarized $F_{1}$ and $F_{2}$ structure functions as

$$
F_{L}=\left(1+\gamma^{2}\right) F_{2}-2 x F_{1}
$$

The $A_{1}$ and $A_{2}$ asymmetries are directly related to the spin structure functions by

$$
A_{1}=\left(g_{1}-\gamma^{2} g_{2}\right) \frac{2 x}{\left(1+\gamma^{2}\right) F_{2}-F_{L}}, \quad A_{2}=\gamma\left(g_{1}+g_{2}\right) \frac{2 x}{\left(1+\gamma^{2}\right) F_{2}-F_{L}}
$$

Since $x$ is bounded by $0 \leq x \leq 1$, at high energies the longitudinally polarized cross section is dominated by the $g_{1}$ structure function, with $A_{1} \rightarrow g_{1} / F_{1}$ in the high- $Q^{2}$ limit, while both $g_{1}$ and $g_{2}$ contribute at the same order to the transversely polarized cross section. At finite $Q^{2}$, however, knowledge of both unpolarized structure functions is necessary for extracting the $g_{1}$ and $g_{2}$ structure functions, and the spin-dependent parton distributions from the inclusive asymmetries.

\section{B. Data}

The inclusive DIS data sets considered in this analysis include polarization asymmetries of the proton, deuteron and ${ }^{3} \mathrm{He}$ measured at CERN in the EMC [1], SMC [30, 31] and COMPASS [32, 33] experiments, at SLAC with the E130 [34], E142 [35], E143 [36], E154 [37], E155 [38-40] and E155x [41] experiments, by the HERMES Collaboration [42-44] at DESY, as well as more recent experiments in Hall A [45, 46] and Hall B [47, 48] at Jefferson Lab.

These data sets are summarized in Table I, which lists the relevant observables available from each experiment, and the number of data points that lie within the $Q^{2}$ and $W^{2}$ cuts. (The data sets can also be found in the online JAM Database [50].) The nominal cuts used

in the JAM analysis are $Q^{2} \geq 1 \mathrm{GeV}^{2}$ and $W^{2} \geq 3.5 \mathrm{GeV}^{2}$. The latter is slightly lower than in some previous spin PDF analyses, and both are significantly smaller than in many unpolarized global PDF analyses, which reflects the more limited range of data available from polarized DIS experiments. In Sec. VII we study the effect on the spin-dependent PDFs of varying these cuts.

For the CERN data [1,30-33], only $A_{1}$ asymmetries are given; since the data are typically taken at high energies, the $A_{2}$ contribution to the measured $A_{\|}$asymmetry is small. The 
TABLE I: Inclusive spin-dependent DIS data sets used in the JAM analysis, indicating the type of asymmetry measured and the number of data points $N_{\text {dat }}$ within the cuts, as well as the contribution of each data set to the overall $\chi^{2}$ of the fits (for the full JAM and leading twist fits). For the HERMES " $n$ " measurement [43], only the extracted "neutron" $A_{1}$ asymmetry is available; for the E155x transverse asymmetries $\tilde{A}_{\perp}$ [41] the target polarizations were not exactly perpendicular to the beam line. The data sets marked with asterisks $\left(^{*}\right)$ are not used in the full JAM fits, but are discussed in Sec. V.

\begin{tabular}{|c|c|c|c|c|c|c|}
\hline experiment & reference & observable & target & $N_{\text {data }}$ & $\chi^{2}(\mathrm{LT}) / N_{\mathrm{dat}}$ & $\chi^{2}(\mathrm{JAM}) / N_{\mathrm{dat}}$ \\
\hline EMC & [1] & $A_{1}$ & $p$ & 10 & 0.42 & 0.39 \\
\hline \multirow[t]{4}{*}{$\mathrm{SMC}$} & [30] & $A_{1}$ & $p$ & 12 & 0.36 & 0.36 \\
\hline & [30] & $A_{1}$ & $d$ & 12 & 1.59 & 1.66 \\
\hline & [31] & $A_{1}$ & $p$ & 8 & 1.37 & 1.35 \\
\hline & [31] & $A_{1}$ & $d$ & 8 & 0.54 & 0.56 \\
\hline \multirow[t]{2}{*}{ COMPASS } & [32] & $A_{1}$ & $p$ & 15 & 0.95 & 0.97 \\
\hline & [33] & $A_{1}$ & $d$ & 15 & 0.57 & 0.51 \\
\hline SLAC E80/E130 & [34] & $A_{\|}$ & $p$ & 23 & 0.52 & 0.54 \\
\hline \multirow[t]{2}{*}{ SLAC E142 } & [35] & $A_{1}$ & ${ }^{3} \mathrm{He}$ & 8 & 0.58 & 0.70 \\
\hline & [35] & $A_{2}$ & ${ }^{3} \mathrm{He}$ & 8 & 0.70 & 0.70 \\
\hline \multirow[t]{4}{*}{ SLAC E143 } & {$[36]$} & $A_{\|}$ & $p$ & 85 & 0.85 & 0.81 \\
\hline & [36] & $A_{\perp}$ & $p$ & 48 & 0.95 & 0.91 \\
\hline & [36] & $A_{\|}$ & $d$ & 85 & 1.05 & 0.85 \\
\hline & [36] & $A_{\perp}$ & $d$ & 48 & 0.92 & 0.91 \\
\hline \multirow[t]{2}{*}{ SLAC E154 } & {$[37]$} & $A_{\|}$ & ${ }^{3} \mathrm{He}$ & 18 & 0.43 & 0.42 \\
\hline & {$[37]$} & $A_{\perp}$ & ${ }^{3} \mathrm{He}$ & 18 & 1.00 & 1.00 \\
\hline \multirow[t]{4}{*}{ SLAC E155 } & [38] & $A_{\|}$ & $p$ & 73 & 1.00 & 0.92 \\
\hline & {$[38,39]$} & $A_{\perp}$ & $p$ & 66 & 1.00 & 0.96 \\
\hline & {$[40]$} & $A_{\|}$ & $d$ & 73 & 0.98 & 0.97 \\
\hline & {$[39,40]$} & $A_{\perp}$ & $d$ & 66 & 1.51 & 1.49 \\
\hline \multirow[t]{2}{*}{ SLAC E155x } & {$[41]$} & $\tilde{A}_{\perp}$ & $p$ & 117 & 2.17 & 1.64 \\
\hline & [41] & $\tilde{A}_{\perp}$ & $d$ & 117 & 0.90 & 0.84 \\
\hline \multirow[t]{4}{*}{ HERMES } & {$[42]$} & $A_{\|}$ & $p$ & 37 & 0.38 & 0.39 \\
\hline & [42] & $A_{\|}$ & $d$ & 37 & 0.86 & 0.85 \\
\hline & [43] & $A_{1}$ & $" n "$ & 9 & 0.29 & 0.30 \\
\hline & [44] & $A_{2}$ & $p$ & 20 & 1.07 & 1.16 \\
\hline \multirow[t]{2}{*}{ JLab E99-117 } & [45] & $A_{\|}$ & ${ }^{3} \mathrm{He}$ & 3 & 0.62 & 0.06 \\
\hline & [45] & $A_{\perp}$ & ${ }^{3} \mathrm{He}$ & 3 & 1.08 & 0.87 \\
\hline COMPASS & [49] & $\Delta g / g$ & $p$ & 1 & 5.27 & 2.71 \\
\hline total & & & & 1043 & 1.07 & 0.98 \\
\hline \multirow[t]{2}{*}{ JLab E97-103* } & [46] & $A_{\|}$ & ${ }^{3} \mathrm{He}$ & 2 & - & - \\
\hline & [46] & $A_{\perp}$ & ${ }^{3} \mathrm{He}$ & 2 & - & - \\
\hline JLab EG1b* & {$[48]$} & $A_{1}$ & $p$ & 766 & - & - \\
\hline (prelim.) & [48] & $A_{1}$ & $d$ & 767 & - & - \\
\hline
\end{tabular}


HERMES $p$ and $d$ data are given in terms of the $A_{\|}$asymmetry [42], while the earlier "neutron" data (extracted from ${ }^{3} \mathrm{He}$ ) are given in terms of $A_{1}$ [43]. The more recent transverse polarization analysis [44] measured the $A_{2}$ asymmetry of the proton. For most of the SLAC experiments on $p$ and $d$ targets, both $A_{\|}$and $A_{\perp}$ were measured; for ${ }^{3} \mathrm{He}$ targets, the earlier E142 experiment [35] presented results for $A_{1}$ and $A_{2}$, while the later E154 experiment [37] provided $A_{\|}$and $A_{\perp}$. For the E155x experiment on $p$ and $d$, the target polarizations were not exactly perpendicular to the beam line, but at an angle, and in this analysis we use the exact kinematics as given in Ref. [41]. Finally, experiments in Hall A at Jefferson Lab with

${ }^{3} \mathrm{He}$ targets obtained the $A_{\|}$and $A_{\perp}$ asymmetries [45, 46], while the Hall B data [47, 48] on $p$ and $d$ targets were given in terms of $A_{1}$. Note that the data from the new EG1b experiment in Hall B [48] supercede the earlier results from the EG1a experiment [47]. However, at present the EG1b data have not yet been fully analyzed to enable them to be used in the full JAM fit, although we will examine the possible effects of the preliminary results on the global fits in Sec. VII.

The inclusive DIS data are supplemented with a model-dependent extraction of the gluon $\Delta g / g$ ratio from semi-inclusive DIS at COMPASS [49]. While this is not ideal, in practice it may be reasonable to include these data since the gluon contribution to the polarization asymmetries only contributes at subleading order (and through QCD evolution) to inclusive DIS. A more detailed analysis of the polarized gluon sea requires data from polarized $p p$ scattering, which will be addressed in a future study [51].

Also listed in Table I are the $\chi^{2}$ values for each of the data sets, as well as the total, for the main JAM fit, as well as from a fit which includes leading twist (LT) contributions only (see Sec. V). Generally, the $\chi^{2}$ values are smaller for the JAM fit, which includes higher twist (HT) and other hadronic and nuclear corrections, than for the fit with leading twist contributions only. The improvement in the overall $\chi^{2} / N_{\text {dat }}$ with the higher twist corrections is in fact quite significant, indicating a clear preference of the data for the presence of higher twist effects.

\section{QCD FRAMEWORK}

In this section we outline the theoretical framework used in the JAM global QCD analysis of spin-dependent PDFs. We begin by summarizing the pertinent results for the structure 
functions in terms of leading twist PDFs at NLO, before discussing our choice of parametric forms for the individual parton distributions and their constraints. We also discuss the treatment of PDF errors, and present a reference fit which will be used as a baseline to study the impact of various hadronic and nuclear corrections in subsequent sections.

\section{A. Structure functions at leading twist}

In the leading twist approximation, the factorization theorems of QCD allow the $g_{1}$ structure function to be expressed in terms of spin-dependent (or helicity) quark and gluon distribution functions. For convenience, we work in moment space, where the $n$-th Mellin moment of a PDF $\Delta f\left(x, Q^{2}\right)(f=q, \bar{q}$ or $g)$ is defined as

$$
\Delta f^{(n)}\left(Q^{2}\right)=\int_{0}^{1} d x x^{n-1} \Delta f\left(x, Q^{2}\right) .
$$

In massless leading twist QCD, the $n$-th moments of the $g_{1}$ structure function can be written as

$$
g_{1}^{(n)}\left(Q^{2}\right)=\frac{1}{2} \sum_{q} e_{q}^{2}\left(\Delta C_{q q}^{(n)}\left(Q^{2}\right) \Delta q^{(n)}\left(Q^{2}\right)+\Delta C_{g}^{(n)}\left(Q^{2}\right) \Delta g^{(n)}\left(Q^{2}\right)\right)
$$

where the moments of the quark and gluon hard scattering coefficient functions $\Delta C_{q q}^{(n)}$ and $\Delta C_{g}^{(n)}$ are calculable perturbatively, and are summarized in Refs. [52, 53] up to NLO.

In the cross sections, or $A_{1}$ and $A_{2}$ asymmetries in Eq. (6), the $g_{2}$ structure function is always suppressed by a power of $\gamma \sim M / Q$. Strictly speaking, therefore, in the massless $\left(Q^{2} \rightarrow \infty\right)$ limit only the $g_{1}$ structure function contributes. If one considers also $g_{2}$, for consistency one needs to include also target mass corrections to $g_{1}$, as we discuss in Sec. V. Furthermore, operators containing masses mix with higher twist operators under renormalization, so that in practice the $g_{2}$ structure function contains twist $\tau=3$ contributions in addition to $\tau=2$. The latter is given through the Wandzura-Wilczek relation in terms of the $\tau=2$ contribution to the $g_{1}$ structure function [54],

$$
g_{2}^{(n)}\left(Q^{2}\right)_{\tau=2}=-\frac{n-1}{n} g_{1}^{(n)}\left(Q^{2}\right)_{\tau=2}
$$

In Sec. V we will also consider higher twist contributions to $g_{2}$, in addition to $g_{1}$. While the $\tau=2$ part of the lowest $(n=1)$ moment of the $g_{2}$ structure function obviously satisfies the 
Burkhardt-Cottingham (BC) sum rule [55],

$$
g_{2}^{(1)}\left(Q^{2}\right)=0,
$$

whether this is also satisfied by the higher twist contributions will be discussed in Sec. V. Note that the leading polarized quark coefficient function $\Delta C_{q q}^{(n)}=1+\mathcal{O}\left(\alpha_{s}\right)$ contributes already at LO, while the polarized gluon enters only at NLO, $\Delta C_{q g}^{(n)}=\mathcal{O}\left(\alpha_{s}\right)$.

\section{B. Parton distributions}

The scale dependence, or evolution, of the polarized PDFs has been calculated up to NLO in perturbative QCD, and has the same structure as in the unpolarized case. We follow closely the formalism adopted in Ref. [56] for the evolution of the Mellin moments of the distributions. In particular, we use the so-called truncated solutions, in which subleading terms are explicitly removed from the solution of the evolution equations. The splitting functions appropriate for the polarized NLO evolution can be found, for example, in Ref. [52].

Since the PDF scale dependence is completely specified by the QCD evolution equations, all parton distributions at any scale are specified by the values of a complete set of distributions at the input scale, for which we have chosen $Q_{0}^{2}=1 \mathrm{GeV}^{2}$. The input distributions at this scale are parametrized as

$$
x \Delta f\left(x, Q_{0}^{2}\right)=N_{f} x^{a_{f}}(1-x)^{b_{f}}\left(1+c_{f} \sqrt{x}+d_{f} x\right) .
$$

for $f=u^{+}, d^{+}, \bar{u}, \bar{d}, \bar{s}, g$, where $q^{+}=q+\bar{q}$ and we assume $\Delta \bar{s}=\Delta s$. The choice of basis functions $\Delta u^{+}$and $\Delta d^{+}$, rather than, say, the valence $\Delta u-\Delta \bar{u}$ and $\Delta d-\Delta \bar{d}$ distributions, is motivated by the fact that these are the functions which naturally enter into the $g_{1}$ structure function in inclusive DIS, see Eq. (8).

In practice, the parametrization (11) is too general for the information available in our analyses, and additional constraints have to be adopted. First, we note that inclusive DIS is only sensitive to three of the quark distributions which, for example, can be taken to be $\Delta u^{+}, \Delta d^{+}$, and $\Delta s^{+}$. The first moments $\Delta q^{+(1)}$ of these distributions are related to matrix elements of weak baryon decays through the relations

$$
\begin{aligned}
\Delta u^{+(1)}-\Delta d^{+(1)} & =1.269 \pm 0.003, \\
\Delta u^{+(1)}+\Delta d^{+(1)}-2 \Delta s^{+(1)} & =0.586 \pm 0.031,
\end{aligned}
$$


These constraints are implemented as additional "data points", so that the fit receives a $\chi^{2}$ penalty if it deviates appreciably from the central values. In practice, in our fits, these latter are very well reproduced with $\chi^{2}$ values close to zero.

Note also that while the distributions are fitted to only two independent observables, $g_{1}^{p}$ and $g_{1}^{n}$ (with the neutron extracted from experiments with deuteron or ${ }^{3} \mathrm{He}$ targets), it is in principle possible to determine three quark PDFs from these because of their different $Q^{2}$ evolution [57]. However, this requires data of sufficient accuracy at different scales, and in practice the constraints obtained from the evolution are not very robust. For example, in fits with a range of (fixed) values for $\Delta s^{+}$, the remaining distributions compensate so that very similar descriptions of the data are achieved in each case. To avoid superfluous parameters (flat directions in $\chi^{2}$ ) and overfitting, we leave only $N_{\bar{s}}$ as a free parameter for $\Delta \bar{s}$, and fix the remaining parameters as $a_{\bar{s}}=a_{d^{+}}, b_{\bar{s}}=b_{g}+2, c_{\bar{s}}=d_{\bar{s}}=0$, and using spectator counting rules at large $x$ [58-60].

Since $\Delta \bar{u}$ and $\Delta \bar{d}$ do not contribute directly to the description of the data in our analysis, these distributions cannot be determined in our fits, and have been fixed by requiring

$$
\lim _{x \rightarrow 0} \Delta \bar{q}\left(x, Q_{0}^{2}\right)=\frac{1}{2} \lim _{x \rightarrow 0} \Delta q^{+}\left(x, Q_{0}^{2}\right)
$$

for $q=u$ and $d$, which implies $N_{\bar{q}}=N_{q^{+}} / 2$ and $a_{\bar{q}}=a_{q^{+}}$. In addition, we choose $b_{\bar{q}}=b_{g}+2$ and $c_{\bar{q}}=d_{\bar{q}}=0$. For our nominal results we have refrained from considering a symmetric sea $(\Delta \bar{u}=\Delta \bar{d}=\Delta \bar{s})$, as has been assumed in some previous analyses [8, 9], since fits that utilize semi-inclusive DIS data [6, 7] typically find a non-symmetric sea — see Fig. 1. (On the other hand, we found that the sea-symmetric assumption provides a comparable description of the data.) In fact, due to the resulting rather flexible parametrization of $\Delta u^{+}$ and $\Delta d^{+}$, the overall size of $\Delta \bar{u}$ and $\Delta \bar{d}$ is poorly constrained. To avoid unphysical results and provide reasonable values for all distributions, we impose in addition the constraints

$$
\frac{1}{2}\left(\left|\frac{\Delta \bar{q}^{(2)}}{\Delta \bar{s}^{(2)}}\right|+\left|\frac{\Delta \bar{s}^{(2)}}{\Delta \bar{q}^{(2)}}\right|\right)=1 \pm 0.25 .
$$

Namely, we enforce that the integrals of $x \Delta \bar{u}$ and $x \Delta \bar{d}$ are comparable to that of $x \Delta \bar{s}$ within a factor of $\approx 2$, although no constraint is imposed on the relative sign of their difference (note that our parametrizations do not allow for nodes in any of these distributions). We stress, however, that the polarized antiquark distributions in the JAM analysis are not fitted directly, but rather determined by the specific choices of parameters outlined above. 
They are considered mostly because of the need for completeness of the set of $Q^{2}$ evolution equations, and should clearly not be viewed as predictions.

As noted in Sec. III A, the gluon distribution $\Delta g$ only contributes directly to the polarized structure functions at a subleading level. In addition, it has some influence on the other distributions through the QCD evolution. The data considered in our analysis provide only mild constraints on this distribution and in fact a good description of most data can be achieved with $\Delta g=0$. Nevertheless, we have allowed a considerable amount of freedom in our fits, including nominally $N_{g}$ and $d_{g}$ as free parameters, with the remaining parameters fixed as $a_{g}=\frac{1}{2}\left(a_{u^{+}}+a_{d^{+}}\right)+1, b_{g}=\frac{1}{2}\left(a_{u^{+}}+a_{d^{+}}\right)+2, c_{g}=0$, in order to obtain a reasonable shape for the gluon distribution.

\section{Statistical estimation and error analysis}

The free parameters of the input distributions have been determined using the formalism detailed in the Appendix of Ref. [61]. This includes a least-squares estimator which takes into account the correlated systematic uncertainties via analytically determined nuisance parameters, and an iterative procedure for an appropriate treatment of multiplicative correlated errors. Although most of the data sets included in our analysis do not provide correlated systematic uncertainties, they do usually include normalizations uncertainties. A proper treatment of these is important in order to avoid different biases which might otherwise occur with more naive treatments (see Ref. [61] for details).

The evaluation of our PDF uncertainties is based on the Hessian method [62]. We have not observed significant tensions between different data sets or encountered particularly flat directions in the parameter space, so that all the free parameters defined in Sec. III B are included in the error calculation. The reported PDF errors in this work refer to variations of $\Delta \chi^{2}=1$ around the minimum. Different choices have also been made in the literature, such as $\Delta \chi^{2}=12.65$ in the AAC analysis [9], while the DSSV group [6] considered both $\Delta \chi^{2}=1$ and $\Delta \chi^{2} / \chi^{2}=2 \%$, and some unpolarized PDF analyses have used even larger values. There is no unique criterion for selecting the correct $\chi^{2}$ interval, and various arguments have been made in favor of different ways to illustrate the effective uncertainty range — see Ref. [9] for a discussion. In the JAM analysis we choose the traditional $\Delta \chi^{2}=1$ interval, based on statistical considerations alone. 


\section{Reference fit}

For our baseline reference fit, we include the same data sets as used in the full JAM fit (listed in Table I), with the same cuts in $Q^{2}$ and $W^{2}$. However, the reference fit uses only leading twist contributions, with no target mass or higher twist corrections, and no nuclear smearing effects. In particular, this means that the $g_{2}$ structure function is approximated by its twist-2 (Wandzura-Wilczek) contribution, Eq. (9). As in the full JAM analysis, we fit proton, deuteron and ${ }^{3} \mathrm{He}$ data directly, rather than the model-dependent experimental extractions of the neutron. For the unpolarized PDF fit, we employ the parametrizations from Ref. [27], which are obtained under similar set of assumptions, kinematic cuts, and theoretical inputs as the JAM fit. The reference fit so constructed allows us to clearly identify the various effects that are introduced in the full JAM analysis.

In Fig. 1 we compare the results of the reference JAM fit for the polarized $\Delta u^{+}, \Delta d^{+}$, $\Delta \bar{u}, \Delta \bar{d}$ and $\Delta \bar{s}$ quark and polarized gluon distributions with several recent parametrizations [6-9] at a scale of $Q^{2}=1 \mathrm{GeV}^{2}$. For clarity, we show the uncertainty bands for the reference and DSSV09 PDFs [6] only, and for the others just the central values. The $\Delta u^{+} \operatorname{PDF}$ is the best constrained polarized distribution, over a relatively broad range of $x$, due mostly to the proton structure function data. The corresponding $\Delta d^{+}$distribution has somewhat larger uncertainties, on the other hand, especially at high $x$ values, since it requires the relatively more scarce ${ }^{3} \mathrm{He}$ (and to a lesser extent deuteron) data. The reference JAM $\Delta u^{+}$ and $\Delta d^{+}$PDFs turn out to be very similar to the DSSV09 and LSS10 distributions, and slightly larger in magnitude at intermediate $x$ compared with the AAC09 and BB10 results. The uncertainty bands for $\Delta u^{+}$and $\Delta d^{+}$are smaller than the total variation between the different parametrizations, which is likely related to the systematic uncertainties associated with the data set choices and theoretical inputs (such as nuclear and finite- $Q^{2}$ corrections) being larger than the experimental uncertainties.

On the other hand, the sea quark polarization is considerably smaller than the total (or valence), with significantly larger errors. Note that while in principle some constraint on the polarized strange distribution $\Delta \bar{s}$ could be obtained from the inclusive DIS data through its $Q^{2}$ evolution, in practice this is challenging because of the limited precision of the $g_{1}$ data. As a result the polarized sea quark distributions are more strongly dependent upon assumptions about flavor symmetry of the proton sea. At present even the semi-inclusive 

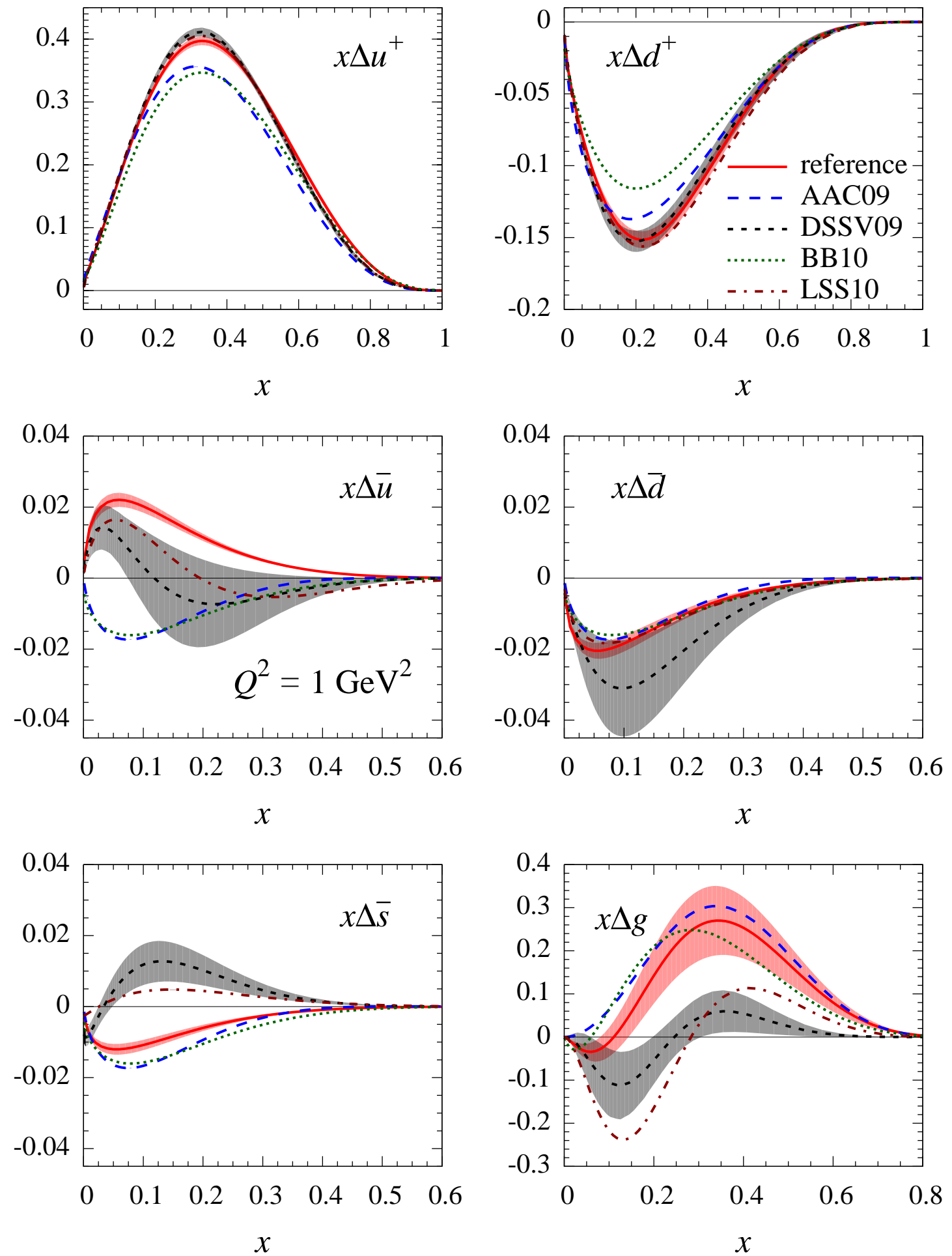

FIG. 1: Spin-dependent parton distributions for the $\Delta u^{+}, \Delta d^{+}, \Delta \bar{u}, \Delta \bar{d}$ and $\Delta \bar{s}$ quark flavors and the polarized gluon $\Delta g$ at a scale of $Q^{2}=1 \mathrm{GeV}^{2}$. The reference JAM fit (red solid) is compared with the recent AAC09 [9] (blue dashed), DSSV09 [6] (black short-dashed), BB10 [8] (green dotted) and LSS10 [7] (brown dot-dashed) parametrizations. 
DIS data do not provide conclusive evidence of a nonzero light quark sea, although a slight trend is indicated towards a more negative $\Delta \bar{d}$ distribution and a positive $\Delta \bar{u}$. In particular, while the $\Delta \bar{d}$ distribution is negative for all the parametrizations shown in Fig. 1, the sign of the $\Delta \bar{u}$ and $\Delta \bar{s}$ PDFs differs for the different fits at intermediate $x$ values. Namely, while the AAC09 and BB10 PDFs, which do not utilize semi-inclusive DIS data, have negative polarized sea distributions, the DSSV09 and LSS10 $\Delta \bar{s}$ parametrizations are positive for $x \gtrsim 0.05$, but negative at smaller $x$. The $\Delta \bar{u}$ distribution is positive at $x \lesssim 0.1-0.2$ for both the DSSV09 and LSS10 fits, but changes signs at larger $x$ values. For the reference JAM fit, the polarized strange PDF is chosen to be negative over all $x$, while $\Delta \bar{u}$ is positive. The signs of the $\Delta \bar{u}$ and $\Delta \bar{d}$ distributions here are in fact determined by those of $\Delta u^{+}$and $\Delta d^{+}$, respectively, with only the sign of $\Delta \bar{s}$ left as a free parameter. The error bands on the JAM antiquark PDFs in Fig. 1 arise from the residual uncertainties on the parameters for the $\Delta q^{+}$distributions, which are related in our analysis to the antiquark PDF parameters [see Eq. (13), for example]. These are not the total uncertainties on the antiquark PDFs, which would need to be determined from a fit to semi-inclusive DIS data. Future measurements of neutral and charged current DIS at an Electron-Ion Collider (EIC) [63-66] will help to clarify the behavior of the polarized sea quarks.

For the polarized gluon distribution, the indirect constraints from the $Q^{2}$ dependence of the inclusive DIS data suggest a positive $\Delta g$, albeit with very large errors, within which the data are also compatible with $\Delta g=0$. This is in contrast with the COMPASS NLO determination of $\Delta g$ from open charm muonproduction, which finds $\Delta g / g=-0.13 \pm 0.15 \pm$ 0.15 at an average $\left\langle Q^{2}\right\rangle \approx 13 \mathrm{GeV}^{2}$ and $\langle x\rangle \approx 0.2$ [49]. This tension results in the larger $\chi^{2}$ values for the COMPASS $\Delta g / g$ point than for all other data sets. On the other hand, the COMPASS extraction of $\Delta g / g$ follows a very specific, model-dependent strategy for the extraction. It will be interesting to explore the consequences of this when data from polarized $p p$ scattering are included in our subsequent analysis [51].

The reference JAM $\Delta g$ is similar in shape to the AAC09 and BB10 fit results, which have a slightly more positive distribution at intermediate $x$ than the DSSV09 and LSS10, which are also more negative at smaller $x, x \sim 0.1$. We note, however, that our polarized gluon is essentially unconstrained except for the normalization and the coefficient of the $(1+x)$ term. Currently most of the information on $\Delta g$ comes from charm production in semi-inclusive DIS at COMPASS [67], and from polarized $p p$ scattering with inclusive pion 
and jet production at RHIC [68,69]. The data are generally consistent with a small value of $\Delta g / g$, consistent with zero, although new measurements from RHIC [4], and possibly a future $\mathrm{EIC}[63,65,66,70]$, have the promise of resolving a small nonzero distribution.

Overall, we obtain a satisfactory fit to the inclusive DIS data with the reference JAM parametrization. This fit will allow us to explore in detail the effects of the various nuclear and hadronic corrections that will be discussed in the following sections. Since the existing inclusive DIS data do not significantly constrain the sea quark and gluon distributions which are dominant at small $x$, we will focus our attention on the polarized valence distributions (or rather the total $\Delta u^{+}$and $\Delta d^{+}$PDFs) at intermediate and high values of $x$.

\section{NUCLEAR CORRECTIONS}

The absence of stable free neutron targets in scattering experiments has required information about the spin structure of the neutron to be obtained using polarized light nuclei, such deuterium or ${ }^{3} \mathrm{He}$, as effective polarized neutron targets. Since the binding energies of these nuclei are small compared with the typical momentum transfers $Q^{2}$, historically the effects of nuclear binding and Fermi motion have been assumed to be negligible. In the static limit, the nuclear effects on the structure functions can be introduced through effective proton $P_{p / A}$ and neutron $P_{n / A}$ polarizations and in the nucleus $A$, with the nuclear $g_{i}^{A}(i=1,2)$ structure functions given by

$$
g_{i}^{A}\left(x, Q^{2}\right)=P_{p / A} g_{i}^{p}\left(x, Q^{2}\right)+P_{n / A} g_{i}^{n}\left(x, Q^{2}\right)
$$

In this effective polarization approximation (EPA) the nuclear effects are therefore assumed to be independent of $x$.

For the case of the deuteron, the two nucleons can exist in either an $S$-state, with relative orbital angular momentum $L=0$, or in a $D$-state, with $L=2$, and share the deuteron spin equally. For most purposes the relativistic $P$-state contributions, with $L=1$, which are associated with negative energy contributions, are negligible [71]. The average polarization of the nucleon $N(p$ or $n)$ is then given by

$$
P_{N / d}=1-\frac{3}{2} \omega_{D}
$$

where $\omega_{D}$ is the the deuteron's $D$-state probability. For realistic models of the deuteron wave function, based on precision fits to $N N$ scattering data, one finds $\omega_{D} \approx 5 \%-7 \%$ [28]. 
For polarized ${ }^{3} \mathrm{He}$ nuclei, most of the time the two protons that accompany the neutron are paired with opposing spins, so that the polarization of ${ }^{3} \mathrm{He}$ is determined mainly by the neutron. The nucleons can be in one of several states, most notably the symmetric $S$-state with a probability $p_{S} \approx 90 \%$, an $L=2 D$-state with probability $p_{D} \approx 10 \%$, and a mixedsymmetric $S^{\prime}$-state with a smaller probability, $p_{S^{\prime}} \approx 1-2 \%$. In terms of these probabilities, the average proton and neutron polarizations in ${ }^{3} \mathrm{He}$ are given by

$$
\begin{aligned}
P_{p / 3} \mathrm{He} & =-\frac{4}{3}\left(p_{D}-p_{S^{\prime}}\right), \\
P_{n /{ }^{3} \mathrm{He}} & =p_{S}-\frac{1}{3}\left(p_{D}-p_{S^{\prime}}\right) .
\end{aligned}
$$

For realistic ${ }^{3} \mathrm{He}$ wave functions computed either by solving the Faddeev equations for the three-body bound state or by using variational methods, the dominant neutron polarization is $\approx 86 \%-89 \%$, with the proton contributing $\approx-4 \%$ to $-6 \%[21,29]$. Note that here $P_{p /{ }^{3} \mathrm{He}}$ is the total proton polarization, rather than the average of the two protons in ${ }^{3} \mathrm{He}$. Higher order corrections to Eqs. (17) from $\boldsymbol{p}^{2}$-weighted moments of the nuclear spectral function, where $\boldsymbol{p}$ is the bound nucleon three-momentum in the nucleus, tend to reduce the magnitude of the neutron polarization by $\approx 1 \%-1.5 \%$, and the proton polarization by $\approx 2 \%-3 \%$ of these values [21].

While the EPA may be a reasonable approximation at low and intermediate values of $x$, at large $x$ where nuclear smearing begins to play a more important role one expects this to break down. In the large- $x$ region the effects of Fermi motion and nuclear binding can be incorporated through convolutions of longitudinally polarized nucleon light-cone distribution functions $f_{i j}^{N / A}$ and bound nucleon structure functions $g_{1,2}^{N}$,

$$
g_{i}^{A}\left(x, Q^{2}\right)=\sum_{N=p, n} f_{i j}^{N / A}(x, \gamma) \otimes g_{j}^{N}\left(x, Q^{2}\right), \quad i, j=1,2
$$

where the convolution $\otimes$ is defined as $(f \otimes g)(x)=\int(d z / z) f(z) g(x / z)$, and the momentum distribution functions $f_{i j}^{N / A}$ in general depend on the light-cone momentum fraction $z$ of the nucleus carried by the active nucleon, as well as on the finite- $Q^{2}$ parameter $\gamma$. Explicit forms for the spin-dependent light-cone distributions (or "smearing functions") $f_{i j}^{N / A}$ have been computed by a number of authors for polarized deuterons [72-76] and ${ }^{3} \mathrm{He}$ [77-79]. Here we shall utilize the smearing functions computed within the weak binding approximation (WBA) by Kulagin et al. for the deuteron [28] and ${ }^{3}$ He targets [29]. Additional corrections 


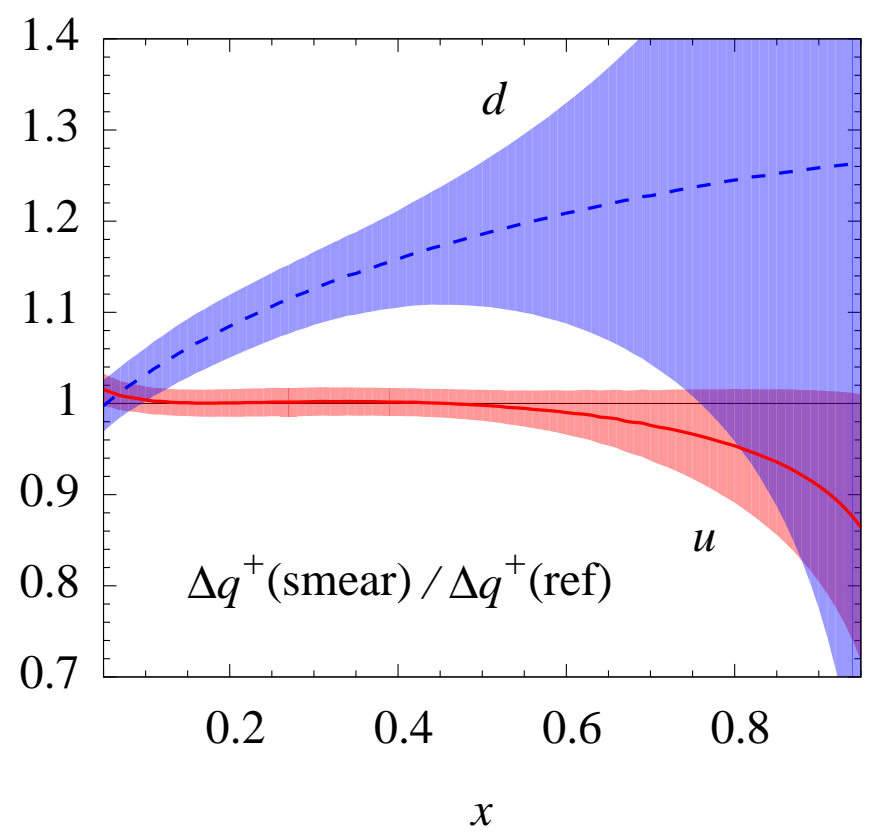

FIG. 2: Ratios of spin-dependent $\Delta u^{+}$and $\Delta d^{+}$distributions, fitted using nuclear smearing corrections, to the reference distributions which use the effective polarization approximation, at a scale $Q^{2}=1 \mathrm{GeV}^{2}$.

to Eq. (18) from the possible modification of the nucleon structure functions in the nuclear medium, as well as from non-nucleonic components of the nuclear wave function, have been considered [21, 80], but are generally expected to be small on the scale of the current experimental uncertainties.

The effects of the nuclear smearing corrections on the $\Delta u^{+}$and $\Delta d^{+}$PDFs are illustrated in Fig. 2, where the ratios of the distributions computed using the smearing functions in Eq. (18) to those approximated by the effective polarization ansatz in Eq. (15) are shown. The $\Delta u^{+}$quark distribution is almost completely inert to the nuclear smearing models over most of the $x$ range, $x \lesssim 0.7$, which reflects the fact that it is determined mainly by the proton data. A $10 \%-20 \%$ suppression is seen at higher $x$ values, although, here the PDFs are essentially unconstrained by data.

A significantly greater impact of the nuclear smearing corrections is visible for the $\Delta d^{+}$ distribution, which is increasingly enhanced for larger values of $x$, reaching $\approx 20 \%-30 \%$ at $x \gtrsim 0.5$ for the central values. In this region the $\Delta d^{+}$distribution is constrained mostly by the polarized ${ }^{3} \mathrm{He}$ data, which at LO is primarily determined by the combination $\Delta u^{+}+$ 
$4 \Delta d^{+}$. The effect of the nuclear smearing correction is to decrease the magnitude of the ${ }^{3} \mathrm{He}$ polarization asymmetry (or $g_{1}$ structure function) at $x \gtrsim 0.5$ [21], resulting in a compensating increase in the magnitude of the (negative) $\Delta d^{+}$distribution in this region. Including the PDF uncertainties, the enhancement can be even larger, ranging from $10 \%-25 \%$ at $x=0.5$ and $\gtrsim 50 \%$ for $x>0.75$. Nuclear smearing corrections are thus vital to take into account if one is to accurately determine the $\Delta d^{+}$distribution at large values of $x$. Similar conclusions have also been reached in connection with the extraction of the unpolarized $d$ quark PDF from deuterium data at high $x$ [20, 22].

\section{FINITE- $Q^{2}$ CORRECTIONS}

The standard global PDF fitting machinery is constructed in order to analyze data in the high- $Q^{2}$ limit, where $Q^{2}$ and $W^{2}$ are both $\gg M^{2}$, away from the region where nucleon resonances or subleading effects in $Q^{2}$ play any significant role. However, if one were to apply the same $Q^{2}$ and $W^{2}$ cuts as are typically employed in unpolarized global PDF analyses [81], much of the spin-dependent DIS data would be excluded. Thus the practical limitations of the polarized DIS data forces us to utilize low- $Q^{2}$ and low- $W^{2}$ data in order to obtain statistically meaningful fits. In this section we discuss in detail various finite- $Q^{2}$ corrections to the formulas for spin-dependent cross sections or structure functions in terms of leading twist PDFs, and examine their impact on the fitted polarized distributions.

In the operator product expansion of QCD, in addition to the usual twist-2 operators $\sim \bar{\psi} \gamma^{\mu} \gamma_{5} \psi$ whose matrix elements give moments of spin-dependent PDFs, there exist operators involving derivatives, such as $\bar{\psi} \gamma^{\mu} \gamma_{5} D^{\gamma_{\mu_{1}}} \cdots D^{\gamma_{\mu_{n}}} \psi$, which are formally also of twist-2, but enter with additional powers of $M^{2} / Q^{2}$. Summing these contributions to all orders in $M^{2} / Q^{2}$, one obtains expressions for structure functions at finite $Q^{2}$ in terms of massless limit $\left(M^{2} / Q^{2} \rightarrow 0\right)$ structure functions and their integrals [82-84]. For the moments of the $g_{1}$ structure function, for instance, one finds [84]

$$
g_{1}^{(n)}\left(Q^{2}\right)=n \sum_{j=0}^{\infty}\left(\frac{M^{2}}{Q^{2}}\right)^{j} \frac{(n+j) !}{j !(n-1) !(n+2 j)^{2}} g_{1(0)}^{(n+2 j)}\left(Q^{2}\right)
$$

where $g_{1(0)}^{(n+2 j)}\left(Q^{2}\right)=\lim _{M \rightarrow 0} g_{1}^{(n+2 j)}\left(Q^{2}\right)$ is the $(n+2 j)$-th moment of the leading twist structure function in the $M^{2} / Q^{2} \rightarrow 0$ limit. 

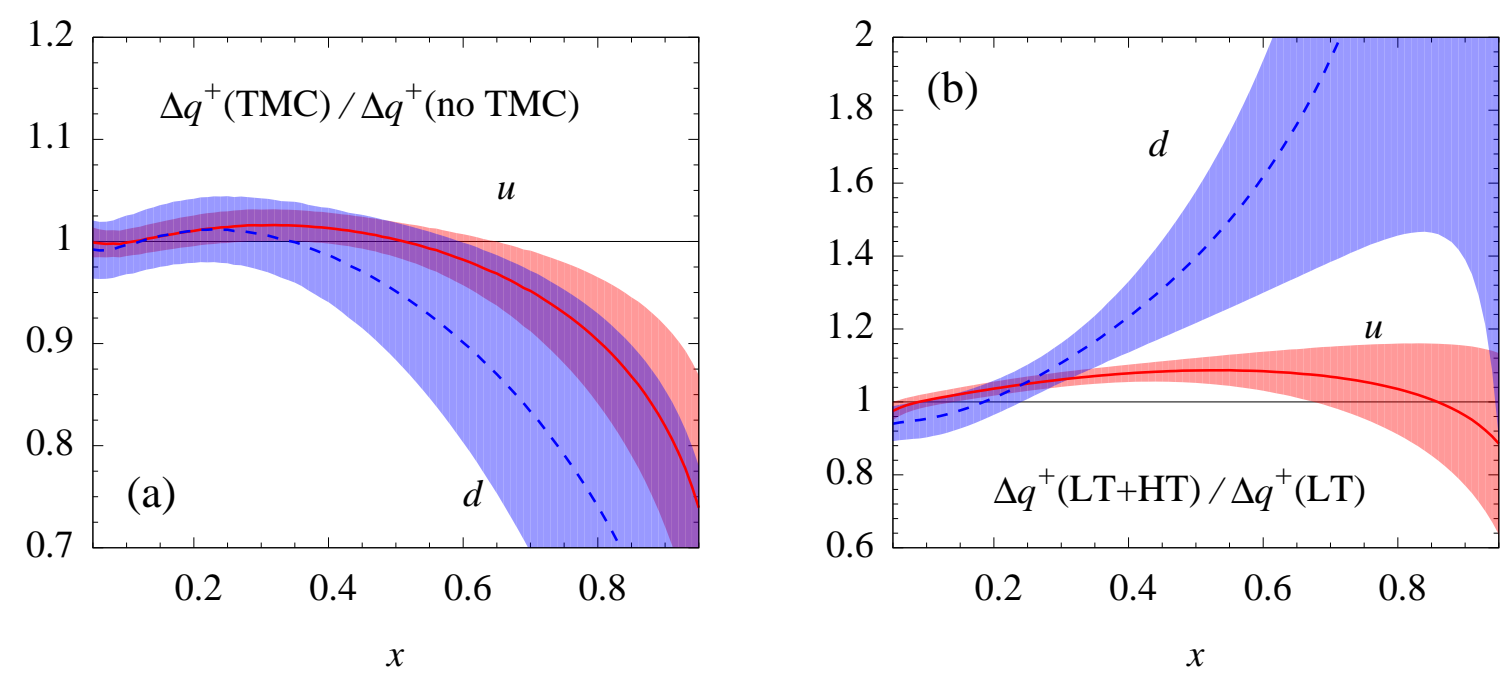

FIG. 3: (a) Ratio of $\Delta u^{+}$and $\Delta d^{+}$distributions, fitted with target mass corrections, to the reference distributions which do not include TMCs. All distributions are fitted incorporating nuclear smearing corrections. (b) Corresponding ratio of $\Delta u^{+}$and $\Delta d^{+}$distributions, computed with higher twist corrections, to the leading twist distributions (nuclear smearing and target mass corrections are included in both fits).

At fixed $Q^{2}$, the effects of TMCs are most significant at large values of $x$. This is illustrated in Fig. 3(a) for the $\Delta u^{+}$and $\Delta d^{+}$distributions, plotted as a ratio to the reference leading twist fit from Sec. IIID at $Q^{2}=1 \mathrm{GeV}^{2}$, which does not include TMCs. The effects of the TMCs increase with increasing $x$, with the distributions including the corrections suppressed relative to the massless limit fit, particularly for the $\Delta d^{+}$PDF. This is consistent with the enhancement of the $g_{1}$ structure function at $x \gtrsim 0.5$ that is generated by the introduction of TMCs [85]; a fit which does not include TMCs will therefore tend to compensate through larger PDFs in this region.

If one includes subleading $1 / Q^{2}$ corrections through the TMCs, then for consistency one must also include contributions from matrix elements of operators with higher twist, $\tau>2$, which enter as $Q^{2}$ power suppressed corrections to cross sections. These typically involve multi-quark and gluon fields and characterize elements of the nonperturbative, long-range correlations among partons. We consider higher twist correction to both the $g_{1}$ and $g_{2}$ 
structure functions,

$$
\begin{aligned}
& g_{1}\left(x, Q^{2}\right)=g_{1}^{(\tau=2)}\left(x, Q^{2}\right)+g_{1}^{(\tau=3)}\left(x, Q^{2}\right)+g_{1}^{(\tau=4)}\left(x, Q^{2}\right), \\
& g_{2}\left(x, Q^{2}\right)=g_{2}^{(\tau=2)}\left(x, Q^{2}\right)+g_{2}^{(\tau=3)}\left(x, Q^{2}\right)
\end{aligned}
$$

with the twist-2 contributions given by Eqs. (8) and (9). The twist-3 contributions to $g_{1}$ and $g_{2}$ are related by the Blümlein-Tkabladze identity [84],

$$
g_{1}^{(\tau=3)}\left(x, Q^{2}\right)=\gamma\left(g_{2}^{(\tau=3)}\left(x, Q^{2}\right)-2 \int_{x}^{1} \frac{d y}{y} g_{2}^{(\tau=3)}\left(y, Q^{2}\right)\right)
$$

For the $\tau=3$ part of $g_{2}$, several parametric forms have been proposed in the literature. For the standard JAM analysis we use a form suggested by the model calculation in Braun et al. [86],

$$
\begin{aligned}
g_{2}^{(\tau=3)}\left(x, Q^{2}\right)= & t_{0}\left(\ln x+1-x+\frac{1}{2}(1-x)^{2}\right) \\
& +\left(t_{1}+t_{2}(1-x)+t_{3}(1-x)^{2}+t_{4}(1-x)^{3}\right)(1-x)^{3},
\end{aligned}
$$

and fit the coefficients $t_{0-4}$ to data.

The parametrization (22) is relatively flexible, and reducing the number of parameters does not substantially change the results. In practice, therefore, the coefficient $t_{4}$ of the highest order $(1-x)$ term is set to zero, $t_{4}=0$. Furthermore, we analytically impose that the BC sum rule (10) is satisfied, leaving us with 3 free parameters. The dependence of the results on the assumptions for the parametrization of the higher-twist contributions to $g_{2}$ will be studied in more detail below.

The twist-4 correction to $g_{1}$,

$$
g_{1}^{(\tau=4)}\left(x, Q^{2}\right)=\frac{h(x)}{Q^{2}},
$$

is determined phenomenologically, using a spline approximation for the $x$ dependence of the numerator $h(x)$. We use knots for the spline at $x=0.1,0.3,0.5$ and 0.7 , with the correction constrained to vanish at $x=0$ and 1 . Note that the form $(22)$ for $g_{2}^{(\tau=3)}$ and the function $h(x)$ in Eq. (23) also neglect the possible $Q^{2}$ dependence associated with TMCs to these twist-3 and twist-4 functions [84], which would contribute at $\mathcal{O}\left(1 / Q^{4}\right)$, as well as with the perturbative $\alpha_{s}$ dependence of the higher-twist matrix elements. (The QCD evolution of the twist-3 contributions to $g_{2}$ was considered in Ref. [87].) This is a reasonable approximation 

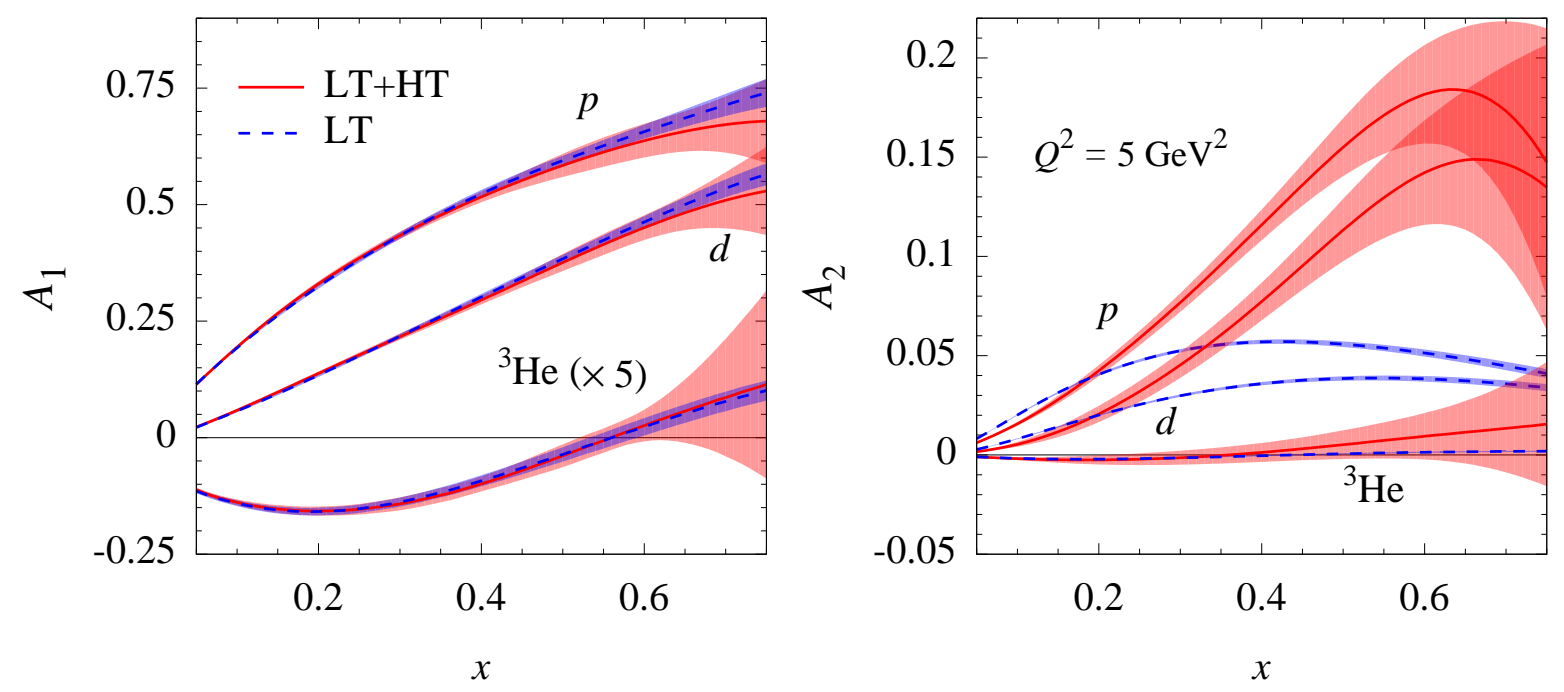

FIG. 4: Polarization asymmetries $A_{1}$ (left) and $A_{2}$ (right) of the proton, deuteron and ${ }^{3}$ He for the JAM fit including leading twist (LT) and higher twist (HT) corrections (red solid), and a fit with LT contributions only (blue dashed), at $Q^{2}=5 \mathrm{GeV}^{2}$. Note that the ${ }^{3} \mathrm{He}$ results for $A_{1}$ are scaled by a factor 5 for clarity.

given the current precision of the polarized DIS data. In practice, the fitted highest twist corrections ( $\tau=4$ term for $g_{1}$ and $\tau=3$ for $g_{2}$ ) also absorb contributions from yet higher order terms in $1 / Q^{2}$, as well as from truncations in the perturbative $\alpha_{s}$ expansion and other unaccounted for effects (such as threshold resummation [88] and jet mass corrections $[89,90])$.

The higher twist corrections have a significant impact on the global fits, as Fig. 3(b) illustrates. While the $\Delta u^{+}$distribution is modified by a modest, $\lesssim 10 \%$ correction, the $\Delta d^{+}$ PDF is strongly enhanced when higher twists are taken into account, by more than a factor of 2 for $x \gtrsim 0.6$. This can be understood by examining the $A_{1}$ polarization asymmetries for the proton, deuteron and ${ }^{3} \mathrm{He}$ in Fig. 4 for the full JAM fit and for the fit which does not include the higher twist corrections. Since the sensitivity to the polarized $d$ quark distribution is greatest for the neutron $g_{1}$ data, the ${ }^{3} \mathrm{He}$ polarization asymmetry provides the strongest constraint on $\Delta d^{+}$. Note that both the full JAM fit and the fit with leading twist only give $A_{1}^{3} \mathrm{He}$ asymmetries (along with the proton and deuteron asymmetries) that are consistent with each other, and describe the data equally well. Any nonzero higher twist component in the full JAM fit would thus have to be offset by an opposing shift in the 

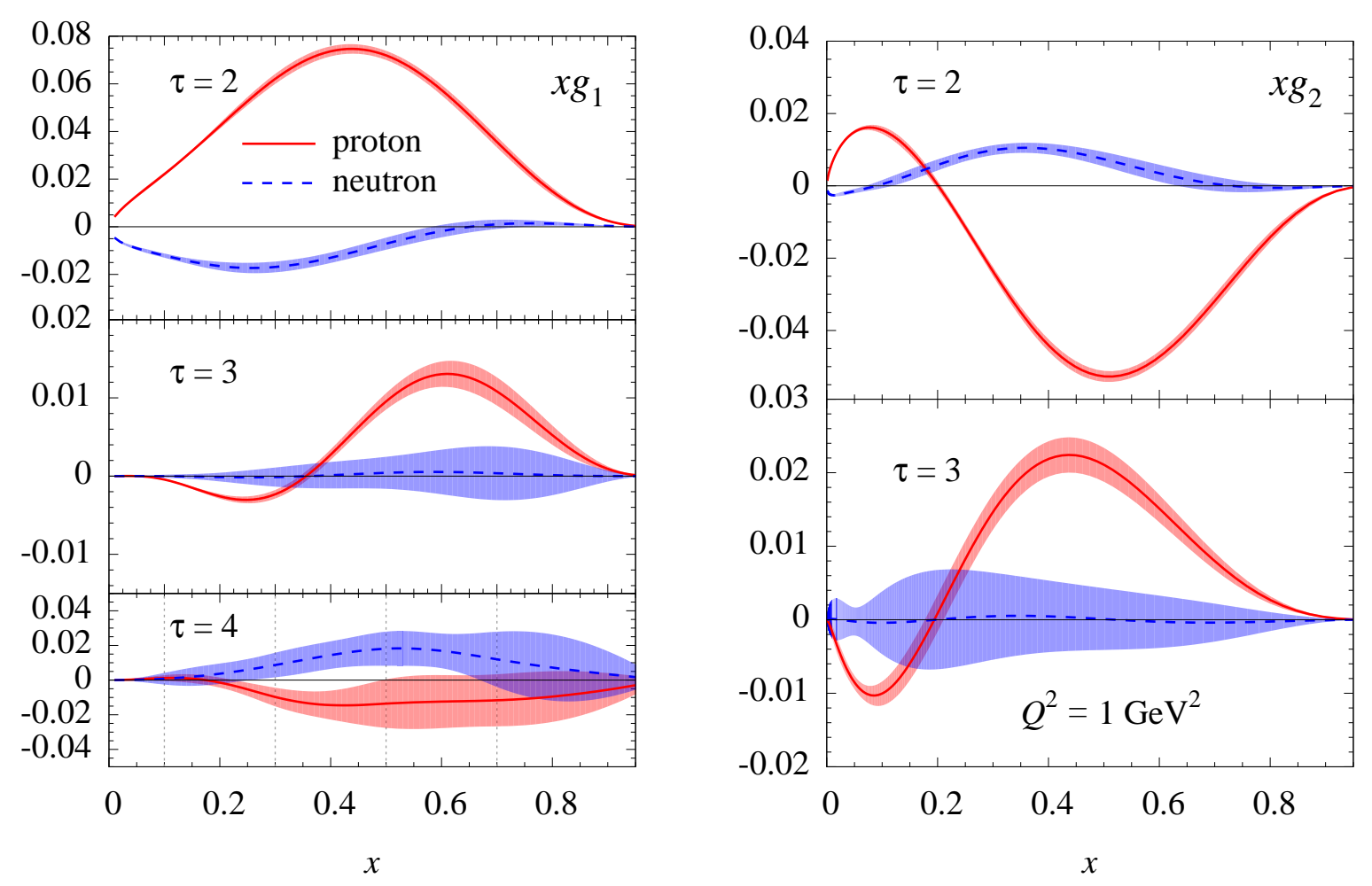

FIG. 5: Twist decomposition of the proton (red solid) and neutron (blue dashed) $x g_{1}$ and $x g_{2}$ structure function. For the $x g_{1}$ structure function (left) the twist $\tau=2$ (top panel), $\tau=3$ (middle) and $\tau=4$ (bottom) are shown, while for the $x g_{2}$ structure function (right) the $\tau=2$ (top) and $\tau=3$ (bottom) contributions are illustrated. The dotted vertical lines on the $\tau=4$ contribution to $x g_{1}$ represent the knots used for the spline fit.

leading twist contribution.

This is indeed observed in Fig. 5, where we present the individual contributions to the $x g_{1}$ and $x g_{2}$ structure functions from the twist-2 and higher twist terms, for both the proton and neutron, at $Q^{2}=1 \mathrm{GeV}^{2}$. For the proton $g_{1}$ structure function, the twist-3 contribution is found to be positive at intermediate $x$ values, $x \gtrsim 0.4$, and negative for the twist- 4 correction, though compatible with zero within the errors. For the neutron, the twist-3 term is consistent with zero, but the twist- 4 contribution is large and positive at $0.3 \lesssim x \lesssim 0.7$. To describe the same experimental ${ }^{3} \mathrm{He}$ asymmetry, the leading twist part of the neutron $g_{1}$ structure function, and hence the $\Delta d^{+}$distribution, must be more negative. The ratio of the (more negative) $\Delta d^{+}$PDF from the full JAM fit to the (less negative) distribution in the leading twist only fit therefore exceeds unity, as seen in Fig. 3(b). 
Of course, the ${ }^{3} \mathrm{He}$ asymmetry depends also on the proton contribution, which is dominated by the $\Delta u^{+}$PDF. However, the partial cancellation of the twist-3 and twist-4 contributions to $g_{1}^{p}$, and the relatively small overall magnitude of the higher twist component compared with the much larger leading twist proton contribution (Fig. 5), means that the impact of the higher twist correction on $\Delta u^{+}$is minimal (Fig. 3(b)).

The leading twist PDFs are of course also indirectly affected by the $g_{2}$ structure functions, as is evident from Eq. (6). For the $g_{2}$ structure function of the proton, we again find a significant positive twist-3 correction at $x \gtrsim 0.2$, which is even larger than the $g_{1}$ correction, and cancels some of the (negative) leading twist contribution. The neutron twist-3 correction to $g_{2}$, on the other hand, is consistent with zero. The large positive $\tau=3$ contribution to the proton $g_{2}$ structure function, which is not $Q^{2}$-suppressed, is also responsible for the strong enhancement of the $A_{2}$ asymmetry for the proton and deuteron seen in Fig. 4, relative to the leading twist asymmetry. For the neutron, the $\tau=3$ correction is consistent with zero, leaving essentially no impact on the $A_{2}$ asymmetry for ${ }^{3} \mathrm{He}$.

The shape of the proton twist-3 correction is similar to that found in the phenomenological analysis in Ref. [91], using the parametrization

$$
g_{2}^{(\tau>2)}\left(x, Q^{2}\right)=\alpha_{0}(1-x)^{\alpha_{1}}\left[\left(\alpha_{1}+2\right) x-1\right]
$$

which was constructed to vanish at $x=1$ and satisfy the BC sum rule (10). The parametrization (24) has a single node, which was found to be located in a similar position as that in the JAM result in Fig. 5, but with a smaller magnitude. The result of the calculation of Braun et al. [86], on the other hand, is similar in magnitude to the JAM result, but of opposite sign at the peak near $x \sim 0.3$. This may be related to the jet mass corrections, which have sizeable contributions to $g_{2}-g_{2}^{(\tau=2)}$ starting already at order $\left(1 / Q^{2}\right)^{0}[90]$.

To study the model dependence of the extracted higher twist correction to $g_{2}$, we perform an additional fit of the data using the functional form in Eq. (24), but with the parameters $\alpha_{0}$ and $\alpha_{1}$ refitted to the extended data used in the JAM analysis. The result of the refit using (24) is remarkably similar to the JAM higher twist correction, for both the proton and neutron, as illustrated in Fig. 6, which suggests that the different magnitude found in Ref. [91] was driven by the input data. As a further check, we fit the data using the full JAM fit form (22), but do not impose the BC sum rule constraint (10). Once again the result is very similar at all values of $x \gtrsim 0.1$, with the unconstrained (negative) proton 

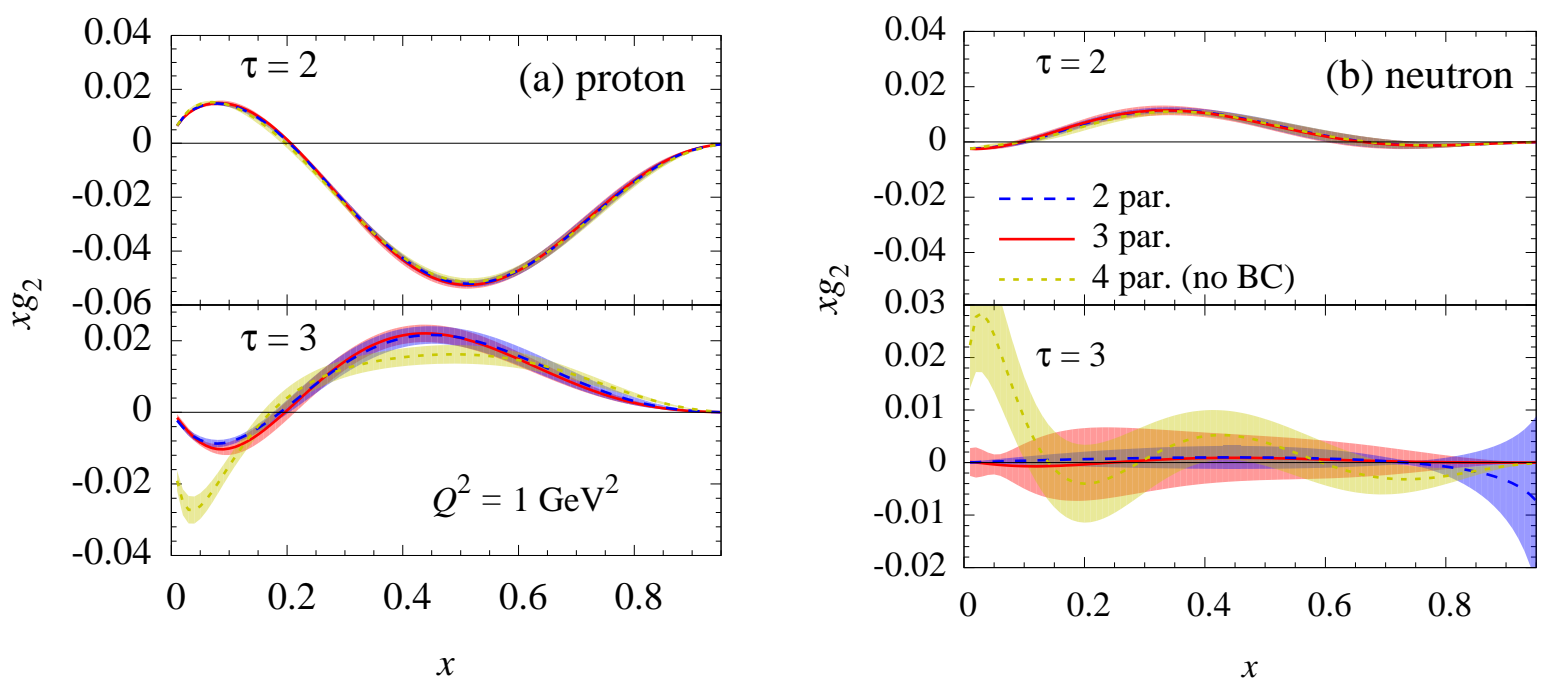

FIG. 6: Contributions to the $g_{2}$ structure function of (a) the proton and (b) the neutron from the twist $\tau=2$ and $\tau=3$ components. The results of the 3-parameter fit (red solid lines) using the functional form in Eq. (22) are compared with the 2-parameter fit (blue dashed lines) of Eq. (24), and with a 4-parameter fit (yellow dotted lines) employing Eq. (22) but without imposing the BC sum rule constraint (10).

correction slightly larger in magnitude at smaller $x$. The unconstrained neutron correction is positive at small $x$, but is compatible with zero at larger $x$ values. The values of the lowest $g_{2}$ moments from the unconstrained fit are found to be $g_{2}^{(1) \text {,fit }}=-0.071 \pm 0.012$ for the proton and $0.092 \pm 0.036$ for the neutron. Overall, this suggests that the extraction of the higher twist contribution to $g_{2}$ is not strongly dependent on the assumed parametrization, and points to a relatively small violation of the BC sum rule.

An additional window on higher twist dynamics is afforded by the $d_{2}$ matrix element of the nucleon, which measures a specific combination of the $n=3$ moments of the $g_{1}$ and $g_{2}$ structure functions, $d_{2}\left(Q^{2}\right)=2 g_{1}^{(3)}\left(Q^{2}\right)+3 g_{2}^{(3)}\left(Q^{2}\right)$. At leading twist this combination of moments vanishes, so that measurement of this matrix elements reveals, to leading order in $1 / Q^{2}$, the twist-3 contribution to $g_{2}$. Our global fits give for the proton $d_{2}$ matrix element $d_{2}^{p}=0.011 \pm 0.002$ at a scale of $Q^{2}=5 \mathrm{GeV}^{2}$, and for the neutron $d_{2}^{n}=0.002 \pm 0.003$. The SLAC E155x experiment measured the $d_{2}$ moments to be [41] $d_{2}^{p(\exp )}=0.0032 \pm 0.0017$ and $d_{2}^{n(\exp )}=0.0079 \pm 0.0048$ at an average $Q^{2}=5 \mathrm{GeV}^{2}$, which are in general agreement with the global JAM values within the uncertainties (note, however, that the $\alpha_{s}$ dependence 
of the twist-3 part of $d_{2}$ is currently not taken into account in the JAM analysis). More recently, the RSS experiment [92] in Jefferson Lab Hall C obtained the contribution $d_{2}^{p(\exp )}=$ $0.0057 \pm 0.0011$ from the resonance region $0.29<x<0.84$ at $\left\langle Q^{2}\right\rangle=1.3 \mathrm{GeV}^{2}$, where the experimental errors have been added in quadrature. Including the extrapolation into the unmeasured region, the inelastic contributions to the proton and neutron moments were found to be [93] $d_{2}^{p(\exp )}=0.0104 \pm 0.0014$ and $d_{2}^{n(\exp )}=-0.0075 \pm 0.0021$ at $\left\langle Q^{2}\right\rangle=1.28 \mathrm{GeV}^{2}$. The contribution from the resonance region to the neutron $d_{2}$ was also recently measured in the E01-012 experiment in Hall A to be [94] $d_{2}^{n(\exp )}=0.0002 \pm 0.0010$ at $\left\langle Q^{2}\right\rangle=2.4 \mathrm{GeV}^{2}$. The E06-014 experiment [95] in Jefferson Lab Hall A is currently analyzing the data on the $d_{2}$ matrix element of ${ }^{3} \mathrm{He}$, from which the neutron value will be extracted at $\left\langle Q^{2}\right\rangle \approx 4 \mathrm{GeV}^{2}$.

\section{JAM FIT RESULTS}

Combining the effects of the nuclear and finite- $Q^{2}$ corrections discussed in the previous sections, the results of the full JAM fits to the data sets in Table I are summarized in Fig. 7. Beginning with the reference parametrizations defined in Sec. IIID, the cumulative effects of the nuclear smearing, target mass and higher twist corrections on the $\Delta u^{+}$and $\Delta d^{+}$ distributions are demonstrated explicitly. (Since the reference fit here is the same as that in Fig. 1, the differences between the JAM fits for $\Delta u^{+}$and $\Delta d^{+}$and the results from previous PDF analyses can also be compared.)

The impact of these corrections is negligible at small values of $x, x \lesssim 0.2$, but grows increasingly important at higher $x$. Compared with the reference distributions, both the JAM $\Delta u^{+}$and $\Delta d^{+}$PDFs are larger in magnitude, by $\sim 10 \%-20 \%$ for the $u$ quark at $0.2 \lesssim x \lesssim 0.6$, and by more than $50 \%-100 \%$ for the $d$ quark at $x \gtrsim 0.4$. The same effects are more clearly illustrated in the form of ratios of polarized to unpolarized PDFs $\Delta u^{+} / u^{+}$ and $\Delta d^{+} / d^{+}$, shown in Fig. 7(b). Such a comparison is meaningful since the unpolarized PDFs are fitted within the same analysis and applied consistently in the extraction of the polarized PDFs.

At intermediate $x$ values, both the $u$ and $d$ quark ratios are broadly consistent with the SU(6) quark model predictions of $\Delta u / u=2 / 3$ and $\Delta d / d=-1 / 3$ [13]. At larger $x$, the $\Delta u^{+} / u^{+}$ratio continues to rise, as expected from helicity conservation models and perturbative QCD calculations [59], which predict that for all quark flavors the ratio $\Delta u^{+} / u^{+} \rightarrow 1$ 

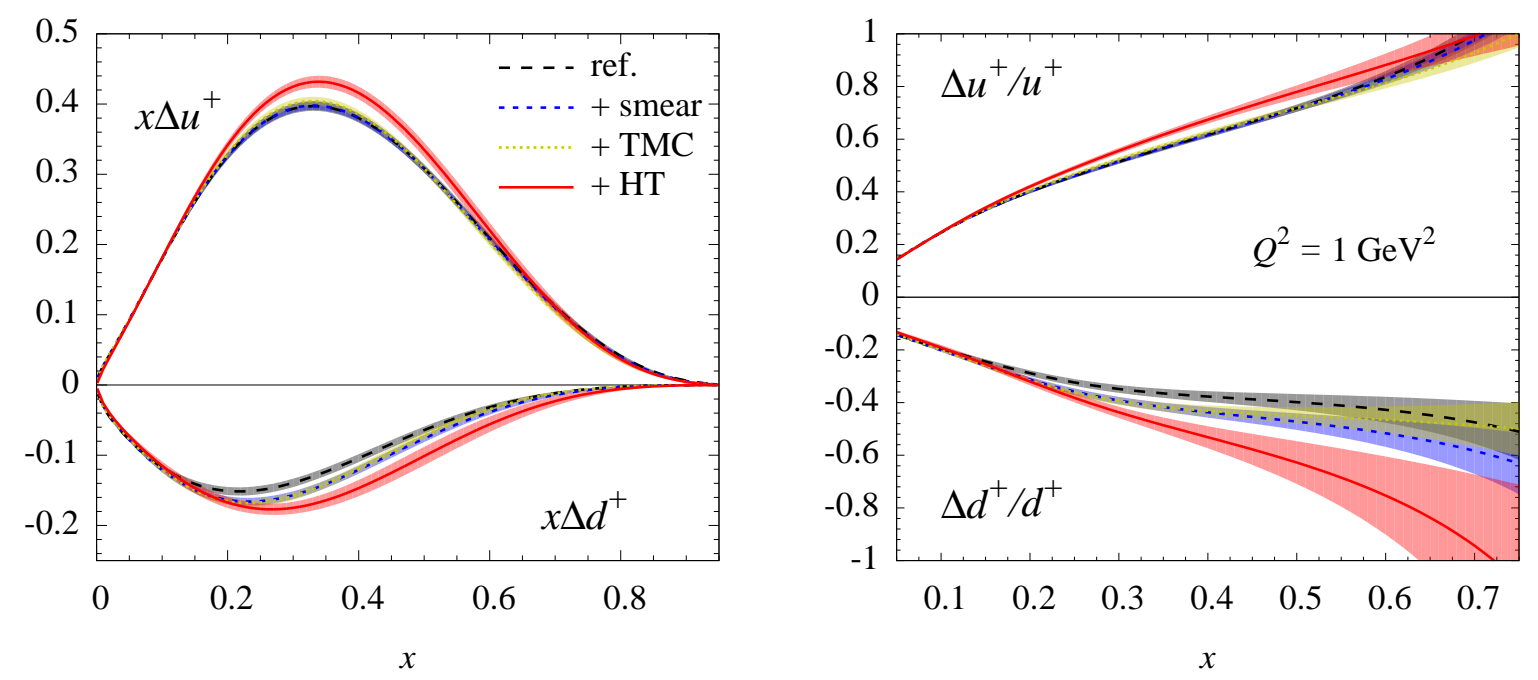

FIG. 7: (Left) Spin-dependent $x \Delta u^{+}$and $x \Delta d^{+}$distributions showing the cumulative effects on the reference PDFs (black dashed line) of adding nuclear smearing (blue short-dashed), target mass (green dotted), and higher twist (red solid) corrections. (Right) Corresponding ratios of polarized to unpolarized $\Delta u^{+} / u^{+}$and $\Delta d^{+} / d^{+}$distributions.

as $x \rightarrow 1$. The $\Delta d^{+} / d^{+}$ratio, however, remains negative with no indication of the upturn predicted by the helicity arguments. Clearly, the nuclear and finite- $Q^{2}$ effects have significantly impact on the asymptotic $x \rightarrow 1$ behavior, and additional data are vital to provide constraints at high $x$.

Although not utilized in the main JAM analysis, additional data at low $Q^{2}$ and low $W^{2}$ do exist from several Jefferson Lab experiments [46, 48, 94, 96], which could affect the large$x$ behavior of the PDFs. In the next section we explore in greater detail the effect on the global fits of these data in order to assess the possible implications of including low- $W$ and low- $Q^{2}$ data in future global analyses.

For completeness, the parameter values for the full JAM fit to the leading twist parton distributions are presented in Table II at the input scale $Q^{2}=1 \mathrm{GeV}^{2}$. The parameters for the twist- 4 correction to the $g_{1}$ structure function in Eq. (23) and the twist-3 correction to the $g_{1}$ structure function in Eq. (23) are listed in Tables III and IV, respectively. In total we find that the inclusive DIS data are able to constrain 13 leading twist parameters, and 7 higher twist parameters each for the proton and neutron. (The fitted parameters in Tables II - IV are given with error bars.) The remaining parameters are constrained using 
TABLE II: Parameter values for the leading twist JAM distributions at the input scale $Q^{2}=$ $1 \mathrm{GeV}^{2}$. The fitted values are those including errors, while the parameters shown without errors are determined through the relations in Sec. III B. Note that the leading twist contributions need to be complemented by the higher twist terms in Tables III and IV.

\begin{tabular}{c|c|c|c|c|c}
\hline \hline flavor $f$ & $N_{f}$ & $a_{f}$ & $b_{f}$ & $c_{f}$ & $d_{f}$ \\
\hline$\Delta u^{+}$ & $1.1167 \pm 0.17$ & $0.8244 \pm 0.04$ & $3.3244 \pm 0.12$ & $-1.8722 \pm 0.30$ & $11.2858 \pm 0.8466$ \\
$\Delta d^{+}$ & $-0.8374 \pm 0.17$ & $0.7193 \pm 0.05$ & $3.9932 \pm 0.40$ & $-1.9324 \pm 0.40$ & $7.0703 \pm 0.7407$ \\
$\Delta g$ & $-0.8120 \pm 0.90$ & 1.7718 & 5.6588 & 0 & $-33.8287 \pm 21$ \\
$\Delta \bar{u}$ & 0.5583 & 0.8244 & 7.6588 & 0 & 0 \\
$\Delta \bar{d}$ & -0.4186 & 0.7193 & 7.6588 & 0 & 0 \\
$\Delta \bar{s}$ & $-0.2073 \pm 0.04$ & 0.7193 & 7.6588 & 0 & 0 \\
\hline
\end{tabular}

TABLE III: Parameter values for the coefficient $h(x)$ of the twist $\tau=4$ contribution to the $g_{1}$ structure function in Eq. (23) at $Q^{2}=1 \mathrm{GeV}^{2}$. The function values are given at the knots $x=0.1$, $0.3,0.5$ and 0.7 .

\begin{tabular}{c|c|c|c|c}
\hline \hline$g_{1}^{(\tau=4)}$ & $h(0.1)$ & $h(0.3)$ & \multicolumn{1}{c|}{$h(0.5)$} & \multicolumn{1}{c}{$h(0.7)$} \\
\hline proton & $0.0118 \pm 0.017$ & $-0.0325 \pm 0.018$ & $-0.0271 \pm 0.028$ & $-0.0167 \pm 0.022$ \\
neutron & $0.0079 \pm 0.034$ & $0.0290 \pm 0.024$ & $0.0362 \pm 0.020$ & $0.0171 \pm 0.023$ \\
\hline
\end{tabular}

TABLE IV: Parameter values for the coefficients $t_{0-4}$ of the twist $\tau=3$ correction to the $g_{2}$ structure function in Eq. (22) at $Q^{2}=1 \mathrm{GeV}^{2}$. The fitted values are shown with error bars.

\begin{tabular}{c|c|c|c|c|c}
\hline \hline$g_{2}^{(\tau=3)}$ & $t_{0}$ & $t_{1}$ & $t_{2}$ & $t_{3}$ & $t_{4}$ \\
\hline proton & -0.0936 & $0.2837 \pm 0.18$ & $0.7542 \pm 0.50$ & $-1.5177 \pm 0.4$ & 0 \\
neutron & -0.0193 & $-0.0136 \pm 0.42$ & $0.1062 \pm 1.25$ & $-0.1456 \pm 1.1$ & 0 \\
\hline
\end{tabular}

the relations in Secs. III B and V. Note that the values in the tables are given to more significant figures than would be appropriate given the quoted error, in order to accurately reproduce our fitted distributions in numerical calculations.

We stress also that the leading twist parameters should not be used to reconstruct the structure functions or polarization asymmetries in isolation of the higher twist contributions. 
Due to the importance of the higher twist corrections in these fits, attempts to reproduce the data, or making predictions for future measurements, with the leading twist distributions alone would lead to discrepancies with experiment.

\section{DATA SELECTION AND CUT SENSITIVITY}

One of the central and novel features of the present JAM global analysis is the inclusion of data over an extended range of kinematics, $Q^{2} \geq 1 \mathrm{GeV}^{2}$ and $W^{2} \geq 3.5 \mathrm{GeV}^{2}$, together with the theoretical corrections needed to reliably account for the nuclear and finite- $Q^{2}$ effects that form an integral part of such an analysis. The cuts themselves are similar to those adopted in some previous analyses; however, the various corrections have not always been applied uniformly. For example, the DSSV analysis [6] applies a cut of $Q^{2}>1 \mathrm{GeV}^{2}$, but extracts the $A_{1}$ asymmetry in Eq. (6) using the approximation $A_{1} \approx A_{1}^{(0)} /\left(1+\gamma^{2}\right)$, where $A_{1}^{(0)}$ is the asymmetry in the massless $\left(M^{2} / Q^{2} \rightarrow 0\right)$ limit, which neglects the $g_{2}$ contribution as well as higher orders in the $1 / Q^{2}$ expansion of the TMCs. The BB fit [8], on the other hand, uses a similar cut in $Q^{2}$ and implements TMCs more systematically, but does not apply nuclear smearing corrections despite using a weaker $W$ cut, $W^{2}>3.24 \mathrm{GeV}^{2}$. In a more recent analysis [97], BB also consider higher twist corrections to $g_{2}$, pointing out the need for $Q^{2}$ evolution of the twist-3 term [86, 87]. As mentioned in Sec. V above, the possible scale dependence of the twist-3 (and twist-4) contributions is not considered in the current JAM fit, but will be included in a subsequent analysis [51].

In this section we investigate the stability of the JAM fits with respect to variations in the $Q^{2}$ and $W^{2}$ cuts from their nominal values, incorporating a priori the full TMC, higher twist, and nuclear smearing corrections. In particular, we consider the effects of varying the $W^{2}$ cut between 3 and $4 \mathrm{GeV}^{2}$, as well as increasing the $Q^{2}$ cut to $2 \mathrm{GeV}^{2}$. In addition, we

perform a leading twist fit utilizing the more stringent cut of $W^{2}>6.25 \mathrm{GeV}^{2}$, as advocated in Ref. [10] to avoid finite- $Q^{2}$ corrections.

Before examining the cut dependence, however, we first consider the effects of including in the global fit data on both longitudinal and transverse asymmetries (or on $A_{1}$ and $A_{2}$ in cases where $A_{\|}$and $A_{\perp}$ are not available). The strategy that is commonly employed in many other analyses of spin-dependent PDFs is to fit $A_{1}$ (or $g_{1}$ ) data extracted from the measured asymmetries while fixing the $g_{2}$ structure function. For example, the standard 

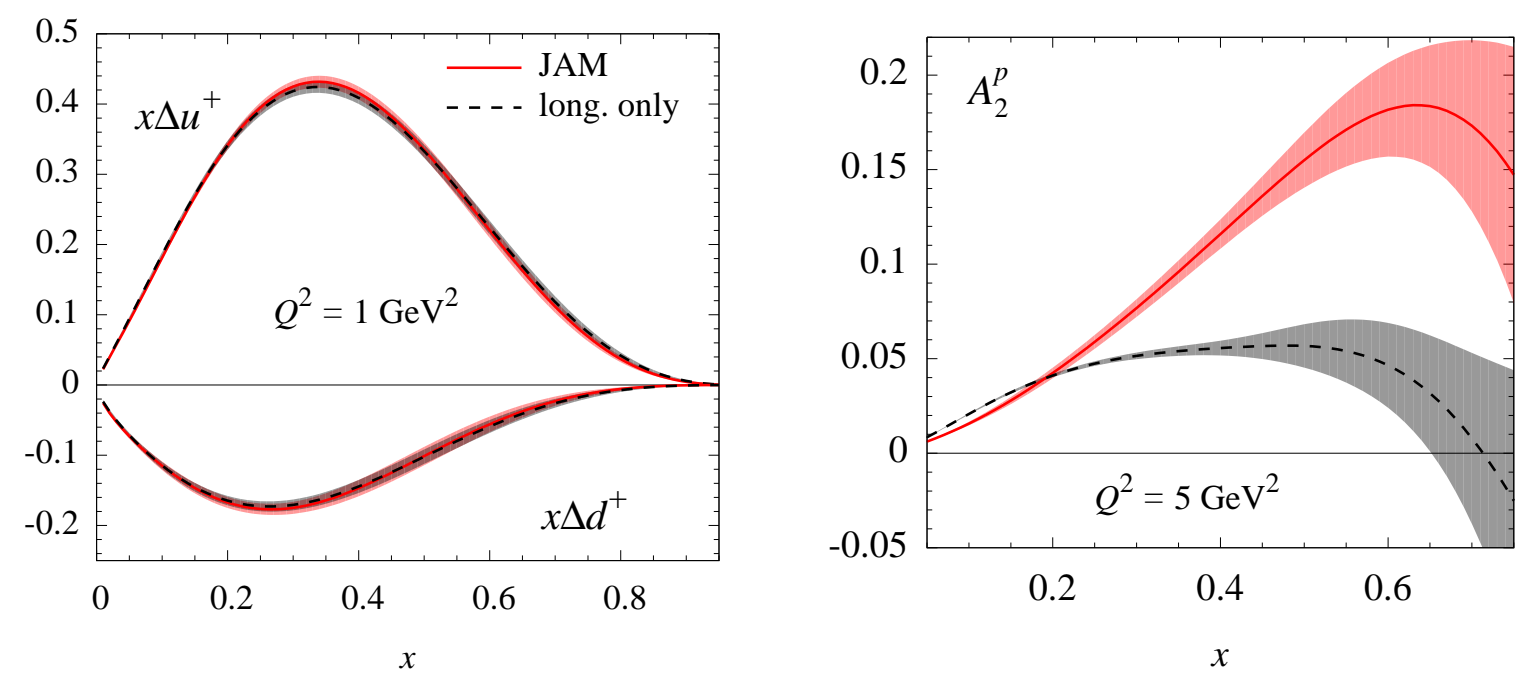

FIG. 8: Comparison of the full JAM fit (red solid lines) with a fit using only longitudinal asymmetries $A_{\|}$(or $A_{1}$ ) (black dashed), for the (left) $x \Delta u^{+}$and $x \Delta d^{+}$distributions at $Q^{2}=1 \mathrm{GeV}^{2}$, and (right) proton $A_{2}^{p}$ asymmetry at $Q^{2}=5 \mathrm{GeV}^{2}$.

assumption is to approximate $g_{2}$ by the twist-2, Wandzura-Wilczek contribution [Eq. (9)], which also implies a vanishing twist-3 contribution to $g_{1}$ [Eq. (21)].

The differences between the full JAM analysis, which simultaneously fits $A_{\|}$and $A_{\perp}$ data, and that based on longitudinal asymmetries only are illustrated in Fig. 8. Since the differences between $A_{1}$ and $A_{\|}$lie mainly (although not exclusively) in the higher twist content of the spin structure functions, the impact on the $\Delta u^{+}$and $\Delta d^{+}$distributions is minimal, with the phenomenological twist-4 correction to $g_{1}$ absorbing most of the differences. On the other hand, the effect on the higher twist contributions to the spin structure functions, particularly $g_{2}$, is significant, as may be anticipated given that longitudinal asymmetries generally receive very small contributions from $g_{2}$. The typical impact of the transverse asymmetry data on the global fits is illustrated in Fig. 8 for the proton $A_{2}$ asymmetry, which shows a signicant (factor $\approx 2-3$ ) enhancement in the full fit at $x \gtrsim 0.4$. One may conclude therefore that while the simultaneous fit gives similar results for the twist-2 PDFs as one based on longitudinal data only, transverse asymmetry data are essential if one is to determine in addition the higher twist content of the structure functions.

The effect of varying the $Q^{2}$ and $W^{2}$ cuts on the $\Delta u^{+}$and $\Delta d^{+}$PDFs is illustrated in Fig. 9 at a scale of $Q^{2}=1 \mathrm{GeV}^{2}$. Compared with the nominal JAM cuts of $Q^{2} \geq 1 \mathrm{GeV}^{2}$ 

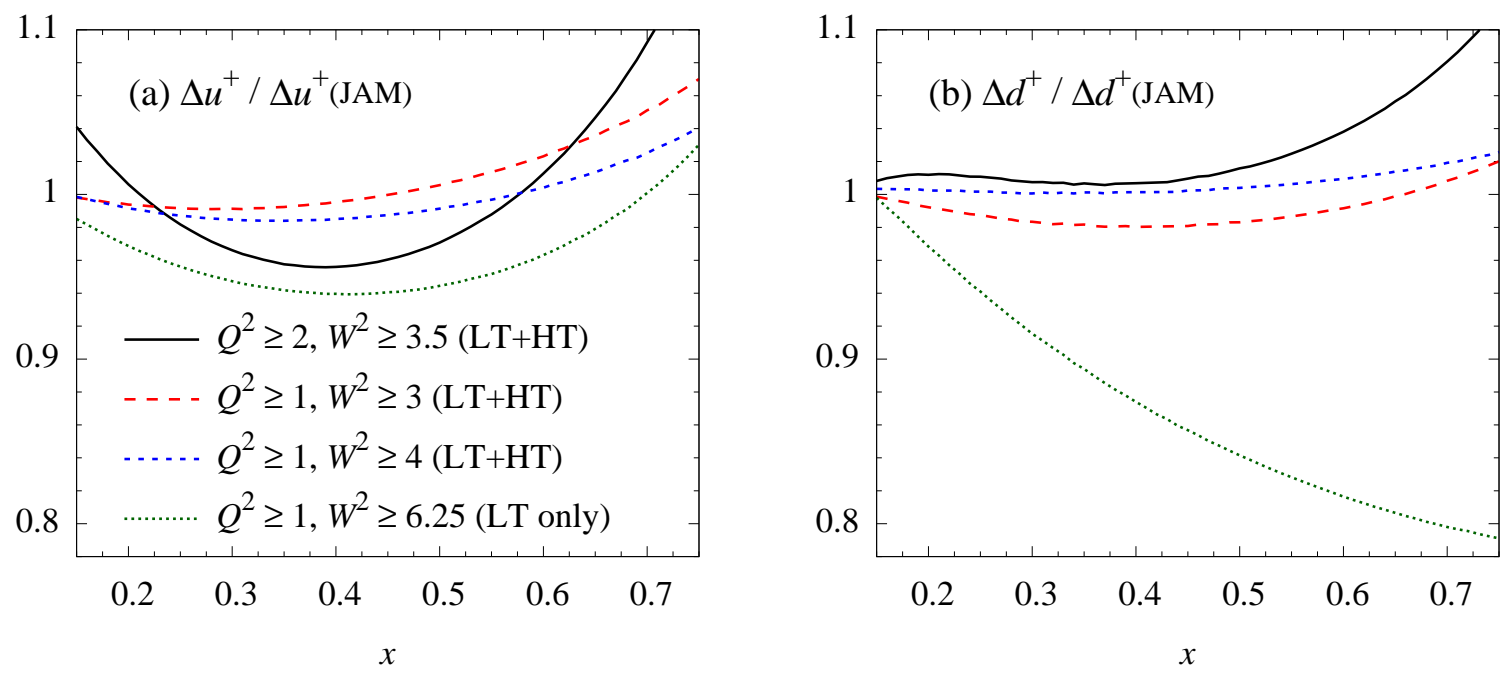

FIG. 9: Ratio of PDFs with various $Q^{2}$ and $W^{2}$ cuts, to the JAM PDFs with the nominal $Q^{2} \geq$ $1 \mathrm{GeV}^{2}$ and $W^{2} \geq 3.5 \mathrm{GeV}^{2}$ cuts, for (a) the $\Delta u^{+}$and (b) the $\Delta d^{+}$distribution. The variations include the cuts $Q^{2} \geq 2 \mathrm{GeV}^{2}, W^{2} \geq 3.5 \mathrm{GeV}^{2}$ (black solid), $Q^{2} \geq 1 \mathrm{GeV}^{2}, W^{2} \geq 3 \mathrm{GeV}^{2}$ (red dashed), and $Q^{2} \geq 1 \mathrm{GeV}^{2}, W^{2} \geq 4 \mathrm{GeV}^{2}$ (blue short-dashed) utilizing LT and HT corrections, and $Q^{2} \geq 1 \mathrm{GeV}^{2}, W^{2} \geq 6.25 \mathrm{GeV}^{2}$ (green dotted) with LT only.

and $W^{2} \geq 3.5 \mathrm{GeV}^{2}$, the variation of the $W^{2}$ cut between $3 \mathrm{GeV}^{2}$ and $4 \mathrm{GeV}^{2}$ has a small, $\lesssim 2 \%$ effect on both the $\Delta u^{+}$and $\Delta d^{+}$distributions, compared with the PDF uncertainties, for most of the $x$ range shown. This suggests that extending the kinematic reach of inclusive DIS data to (marginally) inside the traditional nucleon resonance region $(W \lesssim 2 \mathrm{GeV})$ can still yield stable, leading twist distributions, provided finite- $Q^{2}$ corrections are taken into account. This finding has previously also been observed in global analyses of unpolarized PDFs [20, 22-24]. Qualitatively similar effects are observed when the $Q^{2}$ cut is varied, from 1 to $2 \mathrm{GeV}^{2}$, with $\Delta u^{+}$changing by $\lesssim 4 \%$ for $x<0.6$, and $\Delta d^{+}$by $\sim 1 \%-2 \%$ for $x<0.5$. At higher $x$, both the $\Delta u^{+}$and $\Delta d^{+}$PDFs are enhanced by $\sim 10 \%$ for $x \sim 0.7$, which is outside of the region directly constrained by data. Note, however, that for $W^{2} \geq 3.5 \mathrm{GeV}^{2}$, the restriction to $Q^{2} \geq 1 \mathrm{GeV}^{2}$ means that only the region $x \lesssim 0.28$ is directly constrained by data at this scale, and $x \lesssim 0.43$ for $Q^{2} \geq 2 \mathrm{GeV}^{2}$. In this region the variation in both the $\Delta u^{+}$and $\Delta d^{+}$PDFs is within $\sim 2 \%$ for the different cuts.

Furthermore, exclusion of the $Q^{2}<2 \mathrm{GeV}^{2}$ region means a reduction in the total number of data points by $\approx 50 \%$, since much of the existing polarized DIS data comes from exper- 
iments performed at lower energies than unpolarized experiments. Most global analyses of spin-dependent PDFs therefore choose the $Q^{2} \geq 1 \mathrm{GeV}^{2}$ cut as a practical necessity. As an alternative strategy, the NNPDF Collaboration [10] use data down to $Q^{2}=1 \mathrm{GeV}^{2}$, but impose a more stringent cut of $W^{2}>6.25 \mathrm{GeV}^{2}$ in the expectation that this will allow for an analysis in terms of leading twist contributions only. The impact of this cut on the JAM analysis appears from Fig. 9 to be more dramatic, especially for the $\Delta d^{+}$distribution, which at the $Q^{2}=1 \mathrm{GeV}^{2}$ scale is reduced by $\approx 15 \%$ at $x \approx 0.5$, and by $20 \%$ at $x \approx 0.7$. Such a large difference, together with the results found in Fig. 3(b), reaffirms the necessity of higher twist corrections in analyses which utilize data down to $Q^{2} \approx 1 \mathrm{GeV}^{2}$, as well as the need for new data at higher $Q^{2}$ which can extend the constraints further into the high- $x$ region.

To explore the impact of high- $x$, low- $W$ data on the global PDF analysis, we examine the effect on the spin-dependent PDFs of adding or removing specific data sets. Since the Jefferson Lab Hall A experiment E99-117 [45] provided the most precise data on the ${ }^{3}$ He polarization asymmetries at medium to large $x$ values (up to an average $\langle x\rangle=0.6$ ), we compare the results of the JAM fit, which includes these data, with those obtained by excluding this experiment from the data set. Surprisingly, Fig. 10 indicates that there is almost no difference in the central values of the fitted $\Delta u^{+}$and $\Delta d^{+}$distributions with or without the E99-117 data. There is, however, a visible reduction of the error on the $\Delta d^{+}$ distribution for $x \gtrsim 0.3$ with the inclusion of the E99-117 data, by some $20 \%-25 \%$ at $x=0.6-0.7$.

The E97-103 experiment [46] in Jefferson Lab Hall A also measured the ${ }^{3}$ He polarization asymmetries to very high precision, at an average $x \approx 0.2$ and $Q^{2} \lesssim 1.3 \mathrm{GeV}^{2}$. Although the number of additional data points from this experiment is small (as with the E99-117 experiment), their errors are tiny, which enables them to have a significant impact on the fits in regions that have scant data or where the errors are large. As expected, the effect of the E97-103 data is insignificant for the $\Delta u^{+}$distribution, but gives rise to a $\Delta d^{+}$that is $\sim 10 \%-20 \%$ larger for $x \gtrsim 0.4$. This is clearly an indirect effect of the fits, as the E97-103 data do not constrain this region directly. However, it does reduce the uncertainty on the $\Delta d^{+}$PDF by some $30 \%$ at $x=0.2$ compared with the main JAM fit. Even more strikingly, the E97-103 data places strong constraints on the higher twist part of $g_{2}^{n}$, pinching the error band at $x \approx 0.2$, where the data are taken. It is in fact not possible to fit these data without inclusion of higher twist contributions; doing so would lead to a $50 \%$ reduction in the $\Delta d^{+}$ 

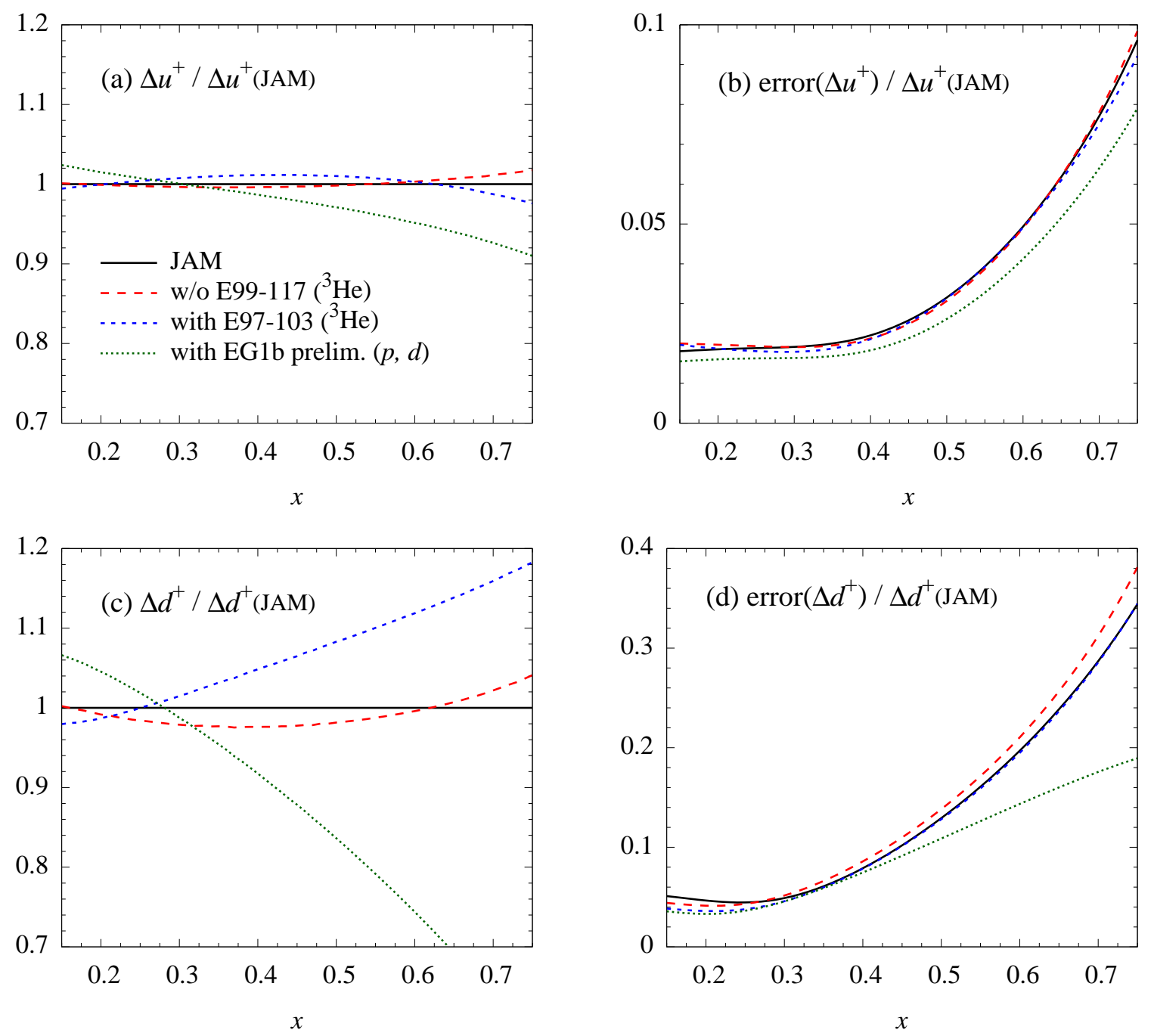

FIG. 10: Effects of various data sets on the $\Delta u^{+}$and $\Delta d^{+}$distributions and their errors, for the full JAM fit at $Q^{2}=1 \mathrm{GeV}^{2}$ with the data sets in Table I (black solid), compared with the fit without the Hall A ${ }^{3} \mathrm{He}$ data in Ref. [45] (red dashed), with the Hall A ${ }^{3} \mathrm{He}$ data in Ref. [46] included (blue short-dashed), and with the preliminary CLAS $p$ and $d$ data in Ref. [48] included. (a) The ratio of the spectator $\Delta u^{+}$distribution to the JAM PDF, together with (b) the relative error with respect to the central values of the JAM PDFs. The corresponding ratios for the $\Delta d^{+}$PDFs are shown in (c) and (d).

distribution, in disagreement with other data.

In contrast, the new data (currently still being finalized) from the EG1b experiment by the CLAS Collaboration at Jefferson Lab [48] contribute over 760 points for $p$ and $d$ over a large kinematic range, up to $Q^{2} \approx 6.5 \mathrm{GeV}^{2}$, albeit with larger errors than for the Hall A 
${ }^{3} \mathrm{He}$ data. Inclusion of these points leads to a small suppression of the $\Delta u^{+}$distribution, which increases at higher $x$, but a significant reduction in $\Delta d^{+}$, by up to $30 \%-50 \%$ for $x$ between $\approx 0.5$ and 0.7 . Concurrently, it also reduces the uncertainty on $\Delta u^{+}$by $15 \%-20 \%$

in the large- $x$ region $(x \gtrsim 0.5)$, and even more significantly for the $\Delta d^{+}$, reducing it by $\approx 30 \%$ at $x=0.6$ and $\approx 50 \%$ at $x=0.7$. This clearly demonstrates the potential impact of the CLAS data for reducing the overall PDF uncertainty at large $x$ values, and will be vital to incorporate in future PDF analyses, once the data analysis is finalized.

\section{CONCLUSION}

We have presented the first results from the JAM global NLO analysis of spin-dependent PDFs from available data on inclusive polarized DIS from protons, deuterons and ${ }^{3} \mathrm{He}$. Where possible, we have fitted directly the measured polarization asymmetries, rather than relying on structure functions extracted under different conditions from the unpolarized cross sections. We include data from all polarized DIS experiments that lie within the limits $Q^{2} \geq 1 \mathrm{GeV}^{2}$ and $W^{2} \geq 3.5 \mathrm{GeV}^{2}$, which allows us to constrain the $\Delta u^{+}$and $\Delta d^{+}$ distributions up to $x \approx 0.7$. Obtaining stable fits over this expanded kinematic range necessitates systematically accounting for target mass and higher twist corrections, which are vital for describing the $g_{1}$ and $g_{2}$ structure functions at the lower $Q^{2}$ range, and nuclear smearing corrections for deuterium and ${ }^{3} \mathrm{He}$ nuclei, which have major impact at large $x$.

The results of the main JAM fit indicate that the $\Delta d^{+}$distribution has a significantly larger magnitude in the intermediate- $x$ region $(x \gtrsim 0.2)$ than in previous analyses, due primarily to the sizeable higher twist corrections found here. In particular, the twist $\tau=3$ term makes important contributions to both the $g_{1}$ and $g_{2}$ structure functions of the proton, and the $\tau=4$ correction makes a large and positive contribution to the neutron $g_{1}$. The latter is mostly responsible for driving the $\Delta d^{+}$distribution to become more negative. The induced twist-3 contribution to the proton $g_{1}$ also reduces the size of the twist-4 term compared to that found previously. The $\tau=3$ correction to the neutron $g_{2}$ is compatible with zero within errors.

The general features of the twist-3 corrections appear to be weakly dependent on the choice of parametrization, except in the small- $x$ region, where some residual differences in their contributions to the $g_{2}$ structure function are found. In particular, parametrizations 
that do not enforce the Burkhardt-Cottingham sum rule yield negative (positive) enhancements at $x \lesssim 0.1$ for the proton (neutron), leading to small violations of the sum rule. On the other hand, differences in the behavior of the structure functions at small $x$ are suppressed for higher moments, such as the $d_{2}$ matrix element. Our global analysis finds a positive contribution to the proton $d_{2}$, of the order $\sim 0.01$ at a scale $Q^{2}=5 \mathrm{GeV}^{2}$, while the neutron $d_{2}$ is consistent with zero. Upcoming data from Jefferson Lab for ${ }^{3} \mathrm{He}$ [95] will better constrain the neutron $d_{2}$. Independent of the details of the extracted higher twists, our analysis clearly highlights the importance of including subleading $1 / Q^{2}$ corrections in any analysis that attempts to fit data down to $Q^{2} \sim 1 \mathrm{GeV}^{2}$, even if more stringent cuts in $W^{2}$ are imposed. In contrast, relatively mild effects are found when varying the $Q^{2}$ and $W^{2}$ cuts in the full JAM analysis including finite- $Q^{2}$ and nuclear smearing corrections.

Future data [18] will also provide additional constraints on the behavior of the ratios $\Delta q^{+} / q^{+}$of polarized to unpolarized PDFs in the $x \rightarrow 1$ limit. This will be important for testing perturbative QCD predictions for the $x \rightarrow 1$ behavior of PDFs, and for exploring the role of orbital angular momentum. While perturbative QCD arguments suggest that the polarized to unpolarized ratio should asymptote to unity at $x=1$ for all quark flavors, our fits for $\Delta d^{+} / d^{+}$show no indication of a rise from its negative value over the currently measured region.

Finally, while the present inclusive DIS data do not substantially constrain the polarized sea quark and gluon distributions at small $x$, in the next phase of the JAM analysis we shall integrate the lessons learned here into an expanded study including semi-inclusive DIS data and hadron production asymmetries in polarized $p p$ scattering, as well as the remaining high-precision data from the completed $6 \mathrm{GeV}$ experiments at Jefferson Lab [48]. This will allow a more robust determination of the polarization of the nucleon sea, and provide a baseline fit which can fully exploit future data from Jefferson Lab at $12 \mathrm{GeV}$, RHIC, the EIC and elsewhere. 


\section{Acknowledgments}

We thank J. Blümlein, H. Böttcher, V. Braun, J.-P. Chen, C. E. Keppel, S. Kuhn, S. Kumano, E. Nocera, O. Rondon, B. Sawatzky and D. Stamenov for helpful comments and discussions. This work was supported by the DOE contract No. DE-AC05-06OR23177, under which Jefferson Science Associates, LLC operates Jefferson Lab. The work of A.A. was supported in part by DOE contract No. DE-SC0008791.

[1] J. Ashman et al., Nucl. Phys. B328, 1 (1989).

[2] S. E. Kuhn, J.-P. Chen and E. Leader, Prog. Part. Nucl. Phys. 63, 1 (2009).

[3] C. A. Aidala, S. D. Bass, D. Hasch and G. K. Mallot, Rev. Mod. Phys. 85, 655 (2013).

[4] P. Jimenez-Delgado, W. Melnitchouk and J. F. Owens, J. Phys. G: Nucl. Part. Phys. 40, 093102 (2013).

[5] E. Leader and C. Lorcé, arXiv:1309.4235.

[6] D. de Florian, R. Sassot, M. Stratmann and W. Vogelsang, Phys. Rev. D 80, 034030 (2009).

[7] E. Leader, A. V. Sidorov and D. B. Stamenov, Phys. Rev. D 82, 114018 (2010).

[8] J. Blümlein and H. Böttcher, Nucl. Phys. B841, 205 (2010).

[9] M. Hirai and S. Kumano, Nucl. Phys. B813, 106 (2009).

[10] R. D. Ball et al., Nucl. Phys. B874, 36 (2013).

[11] C. Bourrely, J. Soffer and F. Buccella, Eur. Phys. J. C 23, 487 (2002).

[12] F. Arbabifar, A. N. Khorramian and M. Soleymaninia, arXiv:1311.1830 [hep-ph].

[13] W. Melnitchouk and A. W. Thomas, Phys. Lett. B 377, 11 (1996).

[14] N. Isgur, Phys. Rev. D 59, 034013 (1999).

[15] R. J. Holt and C. D. Roberts, Rev. Mod. Phys. 82, 2991 (2010).

[16] H. Avakian, S. J. Brodsky, A. Deur and F. Yuan, Phys. Rev. Lett. 99, 082001 (2007)

[17] J. P. Chen, A. Deur, S. Kuhn and Z.-E. Meziani, J. Phys. Conf. Ser. 299, 012005 (2011).

[18] Jefferson Lab Experiments PR12-06-110, J.-P. Chen et al., and PR12-06-122, B. Wojtsekhowski et al., spokespersons.

[19] The Jefferson Lab Angular Momentum (JAM) Collaboration website, http://www . jlab. org/JAM. 
[20] J. F. Owens, A. Accardi and W. Melnitchouk, Phys. Rev. D 87, 094012 (2013).

[21] J. J. Ethier and W. Melnitchouk, Phys. Rev. C 88, 054001 (2013).

[22] A. Accardi, M. E. Christy, C. E. Keppel, P. Monaghan, W. Melnitchouk, J. G. Morfin and J. F. Owens, Phys. Rev. D 81, 034016 (2010).

[23] A. Accardi, W. Melnitchouk, J. F. Owens, M. E. Christy, C. E. Keppel, L. Zhu and J. G. Morfin, Phys. Rev. D 84, 014008 (2011).

[24] S. Alekhin, J. Blümlein, S. Klein and S.-O. Moch, Phys. Rev. D 81, 014032 (2010).

[25] S. Alekhin, J. Blümlein and S.-O. Moch, Phys. Rev. D 86, 054009 (2012).

[26] A. D. Martin, A. J. T. M. Mathijssen, W. J. Stirling, R. S. Thorne, B. J. A. Watt and G. Watt, Eur. Phys. J. C 73, 2318 (2013).

[27] P. Jimenez Delgado and E. Reya, in preparation.

[28] S. A. Kulagin and W. Melnitchouk, Phys. Rev. C 77, 015210 (2008).

[29] S. A. Kulagin and W. Melnitchouk, Phys. Rev. C 78, 065203 (2008).

[30] B. Adeva et al., Phys. Rev. D 58, 112001 (1998).

[31] B. Adeva et al., Phys. Rev. D 60, 072004 (1999); Erratum-ibid. D 62, 079902 (2000).

[32] M. G. Alekseev et al., Phys. Lett. B 690, 466 (2010).

[33] V. Yu. Alexakhin et al., Phys. Lett. B 647, 8 (2007).

[34] G. Baum et al., Phys. Rev. Lett. 51, 1135 (1983).

[35] P. L. Anthony et al., Phys. Rev. D 54, 6620 (1996).

[36] K. Abe et al., Phys. Rev. D 58, 112003 (1998).

[37] K. Abe et al., Phys. Rev. Lett. 79, 26 (1997); Yu. Kolomensky, Ph.D. thesis, U. Massachusetts (1997), SLAC-Report-503.

[38] P. L. Anthony et al., Phys. Lett. B 493, 19 (2000).

[39] P. L. Anthony et al., Phys. Lett. B 458, 529 (1999).

[40] P. L. Anthony et al., Phys. Lett. B 463, 339 (1999).

[41] P. L. Anthony et al., Phys. Lett. B 553, 18 (2003).

[42] A. Airapetian et al., Phys. Rev. D 75, 012007 (2007).

[43] K. Ackerstaff et al., Phys. Lett. B 404, 383 (1997).

[44] A. Airapetian et al., Eur. Phys. J. C 72, 1921 (2012).

[45] X. Zheng et al., Phys. Rev. Lett. 92, 012004 (2004); Phys. Rev. C 70, 065207 (2004).

[46] K. Kramer et al., Phys. Rev. Lett. 95, 142002 (2005); K. Kramer, Ph.D. thesis, College of 
William and Mary (2003).

[47] K. V. Dharmwardane et al., Phys. Lett. B 641, 11 (2006).

[48] Y. Prok et al., Phys. Lett. B 672, 12 (2009); S. Kuhn, private communication.

[49] C. Adolph et al., Phys. Rev. D 87, 052018 (2013).

[50] B. Sawatzky et al., The Jefferson Lab Angular Momentum (JAM) collaboration database, http://www.jlab.org/JAMDataBase.

[51] P. Jimenez-Delgado et al., in preparation.

[52] B. Lampe and E. Reya, Phys. Rep. 332, 1 (2000).

[53] T. Weigl and W. Melnitchouk, Nucl. Phys. B465, 267 (1996).

[54] S. Wandzura and F. Wilczek, Phys. Lett. B 72, 195 (1977).

[55] H. Burkhardt and W. N. Cottingham, Annals Phys. 56, 453 (1970).

[56] P. Jimenez-Delgado and E. Reya, Phys. Rev. D 80, 114011 (2009).

[57] E. Leader, A. V. Sidorov and D. B. Stamenov, Phys. Rev. D 58, 114028 (1998).

[58] R. Blankenbecler and S. J. Brodsky, Phys. Rev. D 10, 2973 (1974).

[59] G. R. Farrar and D. R. Jackson, Phys. Rev. Lett. 35, 1416 (1975).

[60] S. J. Brodsky, M. Burkardt and I. Schmidt, Nucl. Phys. B441, 197 (1995).

[61] P. Jimenez-Delgado, Phys. Lett. B 714, 301 (2012).

[62] J. Pumplin et al., Phys. Rev. D 65, 014013 (2001).

[63] E. C. Aschenauer, R. Sassot and M. Stratmann, Phys. Rev. D 86, 054020 (2012).

[64] E. C. Aschenauer, T. Burton, T. Martini, H. Spiesberger and M. Stratmann, arXiv:1309.5327 [hep-ph].

[65] A. Accardi et al., arXiv:1212.1701 [nucl-ex].

[66] A. Accardi, V. Guzey, A. Prokudin and C. Weiss, Eur. Phys. J. A 48, 92 (2012).

[67] C. Adolph et al., Phys. Rev. D 87, 052018 (2013).

[68] A. Adare et al., Phys. Rev. Lett. 103, 012003 (2009).

[69] L. Adamczyk et al., Phys. Rev. D 86, 032006 (2012).

[70] R. D. Ball et al., arXiv:1310.0461 [hep-ph].

[71] W. Melnitchouk, A. W. Schreiber and A. W. Thomas, Phys. Rev. D 49, 1183 (1994); Phys. Lett. B 335, 11 (1994).

[72] L. L. Frankfurt and M. I. Strikman, Nucl. Phys. A405, 557 (1983).

[73] L. P. Kaptari, K. Yu. Kazakov, A. Yu. Umnikov and B. Kämpfer, Phys. Lett. B 321, 271 
(1994).

[74] W. Melnitchouk, G. Piller and A. W. Thomas, Phys. Lett. B 346, 165 (1995);

G. Piller, W. Melnitchouk and A. W. Thomas, Phys. Rev. C 54, 894 (1996).

[75] S. A. Kulagin, W. Melnitchouk, G. Piller and W. Weise, Phys. Rev. C 52, 932 (1995).

[76] C. Ciofi degli Atti, L. P. Kaptari, S. Scopetta and A. Y. Umnikov, Phys. Lett. B 376, 309 (1996).

[77] C. Ciofi degli Atti, S. Scopetta, E. Pace and G. Salmè, Phys. Rev. C 48, 968 (1993).

[78] R.-W. Schulze and P. U. Sauer, Phys. Rev. C 48, 38 (1993); Phys. Rev. C 56, 2293 (1997).

[79] F. R. P. Bissey, A. W. Thomas and I. R. Afnan, Phys. Rev. C 64, 024004 (2001).

[80] F. R. P. Bissey, V. A. Guzey, M. Strikman and A. W. Thomas, Phys. Rev. C 65, 064317 (2002).

[81] S. Forte and G. Watt, Annu. Rev. Nucl. Part. Sci. 63, 291 (2013).

[82] S. Matsuda and T. Uematsu, Nucl. Phys. B168, 181 (1980).

[83] A. Piccione and G. Ridolfi, Nucl. Phys. B513, 301 (1998).

[84] J. Blümlein and A. Tkabladze, Nucl. Phys. B553, 427 (1999).

[85] A. Accardi and W. Melnitchouk, Phys. Lett. B 670, 114 (2008).

[86] V. M. Braun, T. Lautenschlager, A. N. Manashov and B. Pirnay, Phys. Rev. D 83, 094023 (2011).

[87] V. M. Braun, G. P. Korchemsky and A. N. Manashov, Nucl. Phys. B603, 69 (2001).

[88] G. Sterman, Nucl. Phys. B281, 310 (1987); S. Catani and L. Trentadue, Nucl. Phys. B327, 323 (1989); S. Schaefer, A. Schäfer and M. Stratmann, Phys. Lett. B 514, 284 (2001); G. Corcella and L. Magnea, Phys. Rev. D 72, 074017 (2005).

[89] A. Accardi and J. W. Qiu, JHEP 07, 090 (2008).

[90] A. Accardi and A. Bacchetta, in preparation.

[91] A. Accardi, A. Bacchetta, W. Melnitchouk and M. Schlegel, JHEP 0911, 093 (2009).

[92] F. R. Wesselmann et al., Phys. Rev. Lett. 98, 132003 (2007).

[93] K. Slifer et al., Phys. Rev. Lett. 105, 101601 (2010).

[94] P. Solvignon et al., arXiv:1304.4497 [nucl-ex].

[95] Jefferson Lab experiment E06-014, S. Choi et al., spokespersons.

[96] P. Solvignon et al., Phys. Rev. Lett. 101, 182502 (2008).

[97] J. Blümlein and H. Böttcher, arXiv:1207.3170 [hep-ph]. 